\title{
Impacto de los deterioros neuropsicológicos asociados al consumo de sustancias sobre la práctica clínica con drogodependientes
}

\author{
Verdejo García, A.; López-Torrecillas, f.; Orozco Giménez, C.; Pérez García, M. \\ Departamento de Personalidad, Evaluación y Tratamiento Psicológico. Facultad de Psicología. Universidad de Granada.
}

Enviar correspondencia:

Miguel Pérez García. Departamento de Personalidad, Evaluación y Tratamiento Psicológico. Facultad de Psicología. Universidad de Granada. 18071 Granada. mperez@ugr.es

\section{RESUMEN}

El consumo crónico de diversas drogas (cannabis, heroína, estimulantes) ha sido consistentemente asociado a la presencia de deterioros neuropsicológicos en un amplio espectro de funciones, principalmente: memoria, aprendizaje, atención, concentración y razonamiento. Sin embargo, en los últimos años, la investigación neuropsicológica relacionada con el abuso de sustancias, apoyada en la aparición de tecnologías de nueva generación (neuroimagen funcional, análisis de metabolitos) se ha focalizado en el estudio de posibles alteraciones en las funciones ejecutivas de los lóbulos prefrontales de la corteza, así como su influencia sobre la personalidad, cogniciones y conductas del drogodependiente. El objetivo de nuestra revisión es, por una parte, repasar los principales deterioros neuropsicológicos constatados por los estudios clásicos, así como estos nuevos hallazgos en funciones ejecutivas (planificación, toma de decisiones, control de impulsos), y por otra parte, dado que el estatus neuropsicológico parece tener un papel mediador sobre los resultados del tratamiento, analizar la utilidad e impacto de estos deterioros sobre la práctica clínica con sujetos drogodependientes, considerando factores de especial relevancia, como la potencial reversibilidad de los déficits, la comorbilidad con trastornos de la personalidad y del estado de ánimo y la existencia de anosognosia y déficits específicos de impulsividad. Resaltamos, asimismo, la conveniencia de intervenir específicamente sobre aquellas funciones más directamente asociadas al funcionamiento diario de los drogodependientes, a su perseveración en el consumo y al alto riesgo de recaídas, que podrían ser explicados, al menos parcialmente, por la existencia de alteraciones ejecutivas.

Palabras clave: consumo de drogas, deterioro neuropsicológico, neuroimagen, funciones ejecutivas, reversibilidad.

\section{ABSTRACT}

Chronic consumption of several drugs of abuse (cannabis, heroin, stimulants) has been clearly associated with the presence of neuropsychological impairments in a wide range of functions, mainly: memory, learning, attention, concentration and reasoning. Nevertheless, in the last years, neuropsychological research related to substance abuse, supported by the development of new technologies (functional neuroimaging, metabolite studies) has been focused on the study of possible impairments in the executive functions localized in the cortex prefrontal lobes, and their influence on the substance abuser's personality, cognitions and behaviours. The objective of our review is, first, to summarize the main neuropsychological impairments showed by classic studies and these new discoveries in executive functions, and second, to consider the mediating role of neuropsychological status on treatment outcomes, analyse the usefulness and impact of these impairments on clinical practice with drug addicts, taking into account such particularly relevant factors as the potential recoverability of the impairments, the co-morbidity with personality and mood disorders and the existence of unawareness and specific alterations in impulsivity. We also highlight the convenience of intervening specifically in those functions more relevant to the abuser's ordinary life, his persistence in consumption and the high risk of relapse that could be explained, at least partially, as resulting from the executive impairment.

Key words: substance abuse, neuropsychological impairment, neuroimage, executive functions, recoverability.
E consumo de drogas constituye un fenómeno complejo susceptible de ser abordado desde múltiples enfoques dentro de la Psicología. Desde una perspectiva neuropsicológica, podemos asumir la exis- tencia de una relación bidireccional entre el consumo de drogas y la existencia de deterioros neuropsicológicos. Por una parte, diversos estudios sugieren que la presencia de alteraciones neuropsicológicas subyacentes pue- 
den predisponer al sujeto hacia el consumo; por otra parte, existe una gran cantidad de literatura relacionada con los posibles deterioros neuropsicológicos, asociados a alteraciones conductuales, cognitivas, emocionales y de personalidad, que pueden derivarse del consumo crónico de drogas. En nuestra revisión, nos centraremos en primer lugar en el estudio de aquellos deterioros neuropsicológicos que se han mostrado más consistentemente asociados al consumo de diversas drogas que actualmente presentan altas tasas de prevalencia en las estadísticas de pautas de consumo: cannabis, estimulantes y opiáceos (Informe anual 2000 sobre el problema de la drogodependencia en la Unión Europea), y en segundo lugar, analizaremos el impacto y las posibles implicaciones que pueden derivarse del estudio de los correlatos neuropsicológicos del consumo para la práctica clínica con sujetos drogodependientes.

\section{ALTERACIONES NEUROPSICOLÓGICAS ASO- CIADAS AL CONSUMO DE SUSTANCIAS: ESTA- DO ACTUAL.}

Los estudios clásicos más influyentes sobre alteraciones neuropsicológicas asociadas al consumo de sustancias han mostrado consistentemente la presencia de déficits neuropsicológicos que pueden producirse como consecuencia del consumo de diversas drogas, y que parecen afectar a un amplio espectro de funciones. Sin embargo, las tendencias más actuales de investigación en neuropsicología se han orientado al estudio de posibles deterioros focalizados en las funciones ejecutivas de los lóbulos prefrontales y a la aplicación de diversas técnicas de nueva generación (análisis de metabolitos, neuroimagen funcional) con el objetivo de determinar cómo y dónde ejercen las drogas su influencia neurotóxica en el cerebro.

\subsection{Déficits neuropsicológicos que se han mostra- do consistentemente correlacionados con el consumo de drogas.}

La detección de déficits neuropsicológicos nítidamente asociados al consumo de drogas adictivas supone una tarea muy complicada, debido a la gran cantidad de variables que deben ser consideradas y a las dificultades metodológicas que conlleva su control. La tendencia predominante en la literatura existente es una marcada variabilidad interindividual y temporal, debidas en gran parte a estas consideraciones metodológicas, que incluyen: el tipo de pruebas neuropsicológicas utilizadas, el control de la cronicidad y la severidad del consumo, la supervisión del periodo de abstinencia, los posibles efectos del policonsumo, la amplitud del tamaño muestral o la selección de adecuados grupos control.
El consumo crónico de drogas adictivas parece estar asociado con un perfil de deterioro neuropsicológico común bastante generalizado, sin embargo, debido a la existencia de diferencias significativas en los patrones de enervación, mecanismos de conectividad sináptica y posibles focos de deterioro en función del tipo de sustancia, organizaremos los hallazgos neuropsicológicos registrados por los diversos estudios en función del tipo de droga consumida. Excluimos de nuestro estudio aquellas investigaciones referidas a los deterioros neuropsicológicos derivados del consumo de alcohol, por presentar estos consumidores un perfil de deterioro ampliamente constatado, bien delimitado y asociado a mecanismos cerebrales que difieren de aquellos que intervienen en el deterioro provocado por otras drogas de consumo que actualmente generan mayor desconocimiento, incertidumbre y alarma social.

Lo que presentamos a continuación es, por tanto, una revisión de aquellas funciones que se han mostrado más consistentemente afectadas por el abuso de diversas drogas ilegales de consumo más o menos generalizado: cannabis, estimulantes (cocaína, éxtasis) y opiáceos.

\section{Cannabis (THC)}

La investigación de los deterioros neuropsicológicos asociados al consumo de cannabis (THC) se inició durante la década de los 70. Estos primeros estudios carecían de un control metodológico adecuado y dieron lugar a resultados contradictorios. A partir de los años 80, el incremento del rigor metodológico y el progresivo refinamiento de los diseños experimentales proporcionaron una delimitación mucho más ajustada de los posibles déficits neuropsicológicos que podían derivarse de su consumo.

La investigación de los correlatos neuropsicológicos del consumo de cannabis se ha articulado tradicionalmente en torno a dos tipos de estudios:

(1) aquellos en los que dosis controladas de cannabis son administradas a voluntarios con una historia leve de consumo previo, y (2) estudios naturalistas que analizan el funcionamiento neuropsicológico de consumidores crónicos. Muchos de estos estudios fueron inicialmente llevados a cabo en poblaciones de países en los que el consumo de cannabis estaba ampliamente extendido (India, Egipto y Costa Rica), para después generalizarse a la población de países desarrollados.

Cualquier estudio orientado a detectar posibles déficits neuropsicológicos que pueden derivarse del consumo de cannabis debe discriminar entre (a) aquellos déficits debidos a los residuos psicoactivos de la sustancia sobre el Sistema Nervioso Central (SNC) durante las horas o días que siguen a la intoxicación aguda y (b) aquellos déficits debidos a un efecto tóxi- 
co duradero del cannabis sobre el SNC que persiste incluso una vez que la droga ha abandonado el organismo. Parece claro, por tanto, que los estudios de dosis controlada serán más apropiados y efectivos cuando tratamos de registrar déficits agudos provocados por los efectos de la intoxicación, mientras que los estudios naturalistas nos proporcionarán información acerca de efectos a largo plazo o alteraciones duraderas del SNC.

Entre los primeros estudios, realizados durante la década de los 70 y principios de los 80, y caracterizados fundamentalmente por la ausencia de rigor metodológico y de pruebas neuropsicológicas adecuadas, ninguno de los que utilizaron dosis controladas -ya fueran dosis únicas, dobles o múltiples- consiguieron detectar diferencias significativas entre consumidores y no consumidores de cannabis (Barrat et al., 1972; Cohen et al., 1976; Dornbush et al., 1972; Frank et al., 1976; Jones y Benowitz, 1976), mientras que los estudios naturalistas arrojaban aproximadamente el mismo número de resultados positivos (Wig y Varma, 1977; Mendhiratta et al., 1978 -que detectaron efectos agudos- y Souief (1976) -efectos duraderos sobre el SNC-) que negativos (Culver y King, 1974; Grant et al., 1973; Mendelson et al., 1976). Schaefer et al. (1981) documentaron incluso un funcionamiento intelectual superior entre los sujetos consumidores.

Durante la década de los 80, el progresivo incremento de la calidad metodológica derivó en la constatación de los primeros déficits neuropsicológicos claramente asociados a los efectos del THC sobre el SNC. Varma et al. (1988) Ilevaron a cabo el primer estudio naturalista que supervisaba un periodo controlado de abstinencia de 12 horas previo a la evaluación neuropsicológica. Detectaron déficits en tres de las pruebas utilizadas (pencil tapping, estimación de tamaño y de tiempo). Page, Fletcher y True (1988), en un estudio llevado a cabo en Costa Rica con sujetos que habían consumido durante más de 25 años y con un intervalo de abstinencia no supervisado de entre 12 y 24 horas, mostraron un rendimiento significativamente deteriorado en tareas de atención, procesamiento de información y memoria, además de un funcionamiento social más pobre en comparación con un grupo control igualado en las principales características socio-demográficas. Esta misma muestra fue objeto de un largo estudio prospectivo (Fletcher et al., 1996) incluyendo distintas cohortes de consumidores jóvenes y adultos, en el que se detectaron déficits memorísticos en tareas de recuerdo libre y aprendizaje de listas de palabras, así como en tareas de atención selectiva y dividida.

Previamente, Schwartz et al. (1989) habían identificado deterioros en el rendimiento de los consumidores en el Test de Retención Visual de Benton y en las Historias del WAIS, primero con dos días y después con seis semanas de abstinencia. Block y Ghoneim
(1993) habían identificado daños leves aunque significativos en recuperación memorística, expresión verbal y razonamiento matemático en consumidores crónicos de cannabis después de 24 horas de abstinencia y Solowij et al.(1995) habían identificado sutiles deterioros en el procesamiento atencional asociados a una latencia incrementada del potencial P300, consistente en que los consumidores presentaban problemas en la selección eficiente de información de los estímulos relevantes y en la filtración de material irrelevante. Más recientemente, Ehrenreich et al (1999) informaron de la relación entre déficits en el procesamiento atencional y consumo temprano (anterior a los 16 años) de cannabis.

Estudios experimentales con dosis controladas de cannabis administradas a voluntarios sanos corroboran los deterioros en recuerdo libre y otras funciones memorísticas, atribuido a los efectos de la droga sobre los receptores cannabinoides en el hipocampo (Chait y Pierri, 1992; Heishman, Arasteh y Stitzer, 1996) y a su efecto mediador sobre la memoria a corto plazo, así como sobre funciones atencionales y de coordinación viso-motora. Un estudio de Yuille (1998) en el marco de una investigación acerca de la fiabilidad de la memoria de testigos consumidores incide de nuevo en el deterioro de la Memoria a Corto Plazo, no durante el almacenamiento, sino durante la recuperación.

En resumen, podemos constatar la existencia de un efecto agudo del cannabis sobre diversas funciones neuropsicológicas, sobre todo: atención, recuperación memorística y coordinación viso-motora, entre las 12 y las 24 horas siguientes al consumo, pero hemos de ser mucho más prudentes a la hora de asegurar la existencia de deterioros duraderos en el funcionamiento del SNC debidos a los efectos residuales del THC, ya que estos deterioros suelen ser sutiles, se producen como consecuencia de un consumo crónico y severo y estarían focalizados en atención selectiva y dividida y memoria a corto plazo.

\section{Cocaína}

Los estudios focalizados en la detección de posibles déficits neuropsicológicos provocados por el abuso de cocaína se iniciaron durante la década de los 80, coincidiendo con un incremento masivo de su consumo. Desde entonces, la investigación en el campo ha subrayado el estudio de una serie de funciones que se han mostrado en mayor o menor medida afectadas por el consumo de cocaína: memoria y aprendizaje, atención y concentración y flexibilidad y control mental. Mientras que los estudios pioneros señalaban estas funciones como nítida y consistentemente deterioradas como consecuencia del consumo, estudios posteriores que han considerado los efectos potenciales del policonsumo (sobre todo del consumo 
de alcohol, ya que parece incrementar los efectos neurotóxicos de la cocaína en el cerebro) y que han incorporado muestras más amplias e intervalos de abstinencia prolongados, han venido a delimitar la extensión de estos posibles deterioros y a poner de manifiesto su potencial reversibilidad.

Los resultados de los primeros estudios experimentales sugirieron que el consumo reciente de cocaína provocaba deterioros neuropsicológicos moderados pero significativos en memoria verbal a corto plazo (Ardila et al., 1991; Azrin et al., 1992; Grant y Judd, 1976; Manschreck et al., 1990; Mittenberg y Motta, 1993; O’Malley y Gawin, 1990; Press, 1983; Washton y Gold, 1984), habilidades viso-espaciales (Adamse, 1987), atención (Ardila et al., 1991; Berry et al., 1993; Hoff et al., 1991) y abstracción (Grant y Judd, 1976; O’Malley y Gawin, 1990; Volkow et al, 1988), pero, por otro lado, las habilidades construccionales, la fluidez verbal y la memoria a largo plazo no aparecían afectadas por el consumo (Ardila, Roselli y Strumwasser, 1991). La mayoría de estos estudios fueron diseñados con muestras pequeñas e intervalos breves de abstinencia, por lo que la mayoría de los déficits parecían recuperarse conforme incrementaba el periodo de no consumo.

No obstante, estudios posteriores dotados de un mayor control metodológico continúan señalando la memoria, la atención y las habilidades de razonamiento abstracto como funciones más sensibles al deterioro provocado por el consumo de cocaína. Roselli y Ardila (1996), con una muestra de 108 sujetos cocainómanos y policonsumidores y estrictos criterios de inclusión que requerían un periodo de abstinencia previo a la evaluación de al menos dos meses, consideraron razonable sugerir que la memoria era la función más afectada por el consumo crónico de cocaína, seguida de la abstracción y la atención. Los estudios de Gillen et al. (1998) y Serper et al. (2000) parecen apoyar esta hipótesis al detectar deterioros en funciones memorísticas aún manteniendo intacto el funcionamiento de otros dominios cognitivos. Kouri, Lukas y Mendelson (1996) identificaron déficits de atención en cocainómanos abstinentes. Strickland et al. (1993) mimetizaron el perfil clásico de deterioro al hallar daños neuropsicológicos significativos en tareas que requerían atención sostenida y concentración, memoria verbal y visual, flexibilidad cognitiva y velocidad de procesamiento mental en cocainómanos que habían permanecido abstinentes durante seis meses. Beatty et al. (1995) detectaron deterioros en el rendimiento de los sujetos consumidores en test de aprendizaje, memoria, resolución de problemas, velocidad perceptivo-motora y funciones ejecutivas después de cinco semanas de abstinencia.

Sin embargo, otra serie de estudios han venido a cuestionar, o al menos matizar, los efectos de la cocaína sobre el funcionamiento de la memoria y otras funciones cognitivas. Por ejemplo, Horner (1997), en uno de los pocos estudios que discriminan el deterioro producido por el consumo de cocaína de los efectos del alcohol identificó déficits en medidas de memoria verbal inmediata y demorada, pero no en atención, memoria visuoespacial, habilidades visuoconstruccionales o razonamiento abstracto. Van Gorp et al. (1999) identificaron déficits en el grupo de consumo en una tarea de memoria declarativa (California Verbal Learning Test), pero no en medidas de memoria procedimental (The Pursuit Rotor Task), en la que los sujetos consumidores mostraron un aprendizaje más rápido y duradero. Asimismo, Jasiukaitis y Fein (1999) evidenciaron la no afección de procesos memorísticos implícitos como consecuencia del consumo de cocaína, ni siquiera en aquellos casos en los que se había constatado la existencia de deterioro neuropsicológico severo, tanto los consumidores ligeros como los más deteriorados rendían tan bien como los controles en tareas de priming semántico y de repetición.

Selby y Azrin (1998), en un estudio de riguroso control metodológico, con una muestra de 335 sujetos igualados en cociente intelectual y características socio-demográficas, control del policonsumo y una media de 40.5 meses de abstinencia, no encontraron diferencias significativas en rendimiento neuropsicológico entre consumidores de cocaína y sujetos control en una batería que evaluaba memoria a corto plazo, memoria a largo plazo, habilidades visomotoras y funciones ejecutivas. Asimismo, Beatty y Borrell (2000) mostraron que los sujetos consumidores podían rendir tan bien como los controles en una prueba de memoria que estuviera adaptada al estilo de vida y a las oportunidades de aprendizaje de este tipo de población.

Por tanto, podría afirmarse que la memoria a corto plazo verbal y visual, la atención, la concentración y la flexibilidad y el control mental son las funciones que aparecen más consistentemente afectadas por el consumo crónico de cocaína, si bien (a) los deterioros neuropsicológicos detectados en consumidores de cocaína parecen estar acentuados por el consumo concurrente de otras drogas (alcohol, heroína, benzodiacepinas) y (b) la mayoría de estos deterioros tienden a recuperarse en función de una prolongada abstinencia.

\section{MDMA (Éxtasis)}

Los estudios que investigan los posibles deterioros neuropsicológicos derivados del consumo de MDMA son relativamente recientes y todavía escasos. La proliferación del consumo de MDMA como droga recreativa data de finales de los años 80 y su consumo ha estado normalmente restringido a poblaciones y marcos espacio-temporales muy específicos (fines de semana, grandes discotecas) que han complicado las 
posibilidades de efectuar estudios experimentales metodológicamente controlados. No obstante, la investigación disponible ha puesto de manifiesto el enorme potencial neurotóxico de esta droga, capaz de provocar deterioro neuropsicológico prácticamente desde el inicio de sus consumo, y su capacidad para afectar a diversas áreas de la personalidad del sujeto consumidor.

Diversos estudios parecen indicar que el consumo recreativo de MDMA puede inducir déficits en memoria con otras funciones cognitivas relativamente preservadas (McGuire, 2000). Kristal y Price (1992) hallaron deterioro de la memoria inmediata y demorada en la Escala de Memoria de Weschler, mientras que Morgan (1998) identificó deterioro de esas mismas funciones en las tareas de la Ribermead Behavioral Memory Test. Parrot et al. (1998) compararon tres grupos de diez sujetos, clasificando como consumidores regulares a aquellos que habían tomado éxtasis en diez ocasiones o más, los consumidores novatos eran aquellos que habían consumido éxtasis entre una y nueve veces y los controles no habían consumido nunca. Los resultados mostraron que el uso recreativo de MDMA no estaba asociado con alteraciones en la integridad del sistema de procesamiento básico, pero sí con deterioros en memoria verbal inmediata y demorada en ambos grupos consumidores. Bolla et al. (1998) replicaron estos resultados en memoria visual inmediata y demorada.

McKetin y Mattick (1998) observaron deterioros leves en sujetos clasificados como dependientes (Severity of Dependence Scale; Gossop et al., 1995) en medidas de memoria verbal, atención-concentración y recuerdo demorado de la WMS-R, pero no se observaron déficits en usuarios no dependientes. La existencia de déficits de concentración en consumidores recreativos de MDMA había sido previamente constatada por Solowij (1992).

Por otro lado, Wareing, Fisk y Murphy (2000), utilizando una tarea de generación aleatoria de letras (Baddeley, 1996) sensible al componente "ejecutivo central" de la Working Memory, mostraron que usuarios recreativos de MDMA generaban menos letras y exhibían un mayor grado de redundancia y mayor número de intrusiones, resultados que apoyan la hipótesis de Morgan (1998) en torno a la incapacidad de estos sujetos para afrontar niveles elevados de demanda cognitiva y ponen de manifiesto la afectación del componente ejecutivo de la memoria como consecuencia del consumo.

Asimismo, diversas alteraciones de la personalidad y síntomas psiquiátricos persistentes han sido asociados al consumo de MDMA (Creighton et al., 1991; McCann y Ricaurte, 1991; McGuire y Fahy, 1991; Schifano, 1991).
En conclusión, podemos afirmar (a) que, a diferencia de otras drogas en las que determinados parámetros de cronicidad y severidad son necesarios para que se produzca deterioro neuropsicológico, el consumo esporádico de MDMA con fines recreativos puede generar déficits neuropsicológicos significativos en funciones memorísticas, ejecutivas y de personalidad, y (b) que los efectos potenciales de su consumo prolongado sobre el funcionamiento cerebral a largo plazo permanecen aún sin dilucidar.

\section{Opiáceos}

En comparación con el cannabis y los estimulantes, ha existido una sustancialmente menor cantidad de investigación acerca de los posibles déficits neuropsicológicos asociados al consumo de opiáceos. La investigación de estos potenciales deterioros se desarrolló extraordinariamente en EEUU durante la década de los 70, vinculada a la convulsión social que provocaron los múltiples casos de trastornos de ansiedad y brotes psicóticos presuntamente asociados al consumo de heroína en antiguos combatientes de Vietnam (Fields y Fullerton, 1975) y decayó progresivamente en función de la reducción de las tasas de prevalencia de su consumo a favor del consumo de otras drogas.

El perfil neuropsicológico de los consumidores de opiáceos incluye, a corto plazo, déficits en memoria verbal y visual demorada, atención y concentración, habilidades motoras finas, habilidades viso-espaciales y viso-motoras y fluencia verbal, y a largo plazo, deterioro en funciones ejecutivas y razonamiento abstracto.

Entre los déficits a corto plazo, en general, los consumidores de opiáceos parecen más probablemente dañados en baterías neuropsicológicas tradicionales como la Halstead-Reitan, la Weschler Adult Intelligence Scale (WAIS) y los Test de Afasia (Grant et al., 1978). Hill y Mikhael (1979) encontraron evidencia de daño en algunas funciones de memoria y velocidad motora fina, pero no en tareas que implicaban abstracción y razonamiento. Rodríguez-Álvarez (1981) identificó daños en memoria visoconstructiva inmediata utilizando el Test de Retención visual de Benton. Hill et al. (1979) encontraron que la cronicidad del consumo de opiáceos correlacionaba moderadamente con la cantidad y magnitud del daño cognitivo. En estudios más recientes, Hanks et al. (1995) hallaron deterioros en medidas de memoria verbal y visual demorada, atención y concentración, y Kouri, Lukas y Mendelson (1996) identificaron patrones disfuncionales del potencial P300 en consumidores que habían permanecido abstinentes durante 15 días.

Respecto a los déficits a largo plazo, las líneas de investigación más recientes ubican los deterioros consecuentes al consumo de opiáceos en las funciones ejecutivas del lóbulo prefrontal (Madden et al., 1997; Rogers et al., 1999), pese a que estudios previos habí- 
an descartado la afectación de estas funciones (Bruñí y Maage, 1975; Rounsaville et al., 1982).

Queda por determinar, asimismo, la pervivencia y potencial reversibilidad de los déficits provocados por el consumo de opiáceos, ya que estudios como el de Gerra et al. (2000), no detectan diferencias significativas entre los perfiles neuropsicológicos de consumidores y no consumidores de heroína después de cuatro meses de abstinencia.

En conclusión, podemos afirmar (a) que los sujetos consumidores de heroína presentan un amplio espectro de déficits neuropsicológicos a corto plazo; (b) que a largo plazo las funciones más consistentemente afectadas son el razonamiento abstracto y las funciones ejecutivas.

\subsection{Nuevos hallazgos en Funciones Ejecutivas.}

Los síntomas clave de la drogadicción en humanos son la ingesta compulsiva y el intenso impulso hacia el consumo a expensas de otras conductas que pueden resultar más ventajosas a medio o largo plazo (APA, 1994). Si estructuramos la conducta anormal característica del drogodependiente en distintos componentes, tendríamos (a) un componente de expectativa: basado en las predicciones de recompensa y en la atribución de propiedades probabilísticamente reforzantes al estímulo (droga); (b) un componente de "drive" compulsivo: que es un estado motivacional; y (c) un tercer componente de toma de decisiones: que se basa tanto en las propiedades motivacionales del estímulo como en la importancia relativa concedida a la expectativa de recompensa inmediata frente a posibles pérdidas a largo plazo. Los déficits exhibidos por sujetos drogodependientes en estos tres procesos llevaron a inferir la existencia de un posible deterioro neuropsicológico en las funciones ejecutivas localizadas en los lóbulos prefrontales del córtex.

La investigación de estos deterioros en funciones ejecutivas ha sido consistentemente ignorada en los estudios clásicos del campo, debido posiblemente a la ausencia de modelos teóricos que explicaran de forma exhaustiva el comportamiento adictivo, y a la escasez de pruebas neuropsicológicas sensibles al daño específico en estas funciones. Sin embargo, en los últimos años, diversas líneas de investigación, al amparo de nuevos modelos teóricos (Bechara, Damasio y Damasio, 2000; London et al., 2000), han abordado el estudio de los deterioros neuropsicológicos en funciones ejecutivas asociados al consumo crónico de drogas, utilizando diversos procedimientos de evaluación originales. Por este motivo, estos nuevos hallazgos en funciones ejecutivas merecen especial mención en nuestra revisión. Puesto que estos trabajos no son aún muy numerosos, y en su mayoría abarcan el estudio de distintas sustancias de consumo: cannabis, cocaína, anfetaminas y opiáceos, y asumiendo además que los mecanismos psicoactivos y potencialmente neurotóxicos de todas estas sustancias convergen en las áreas cerebrales objeto de estudio, no realizaremos distinciones en función del tipo de droga de consumo.

Rogers et al. (1999) realizaron una de las investigaciones pioneras dentro de este nuevo paradigma. En su estudio, compararon la conducta de toma de decisiones de consumidores crónicos de anfetaminas y opiáceos con la de pacientes que sufrían lesiones focales orbitofrontales y dorsolaterales. Sus resultados mostraron que ambos grupos de consumidores mostraban incrementos temporales en los periodos de deliberación similares a los observados en pacientes orbitofrontales, pero no en pacientes dorsolaterales. Asimismo, los pacientes orbitofrontales y los consumidores de anfetaminas mostraron una marcada tendencia a elegir la menos ventajosa de las opciones, sugiriendo un empobrecimiento de la calidad de la toma de decisiones, que en el caso de los consumidores de anfetaminas estaba negativamente correlacionado con la cronicidad del abuso. Ambos grupos parecían compartir una tendencia a tratar de obtener recompensa a través de la menos favorable de las opciones disponibles. Estos deterioros fueron mimetizados en sujetos normales voluntarios mediante una reducción dietética de los niveles de triptófano.

Ornstein et al. (2000) examinaron los perfiles de disfunción neuropsicológica en consumidores crónicos de anfetaminas y heroína comparándolos con sujetos controles en una batería computerizada (CANTAB battery; CeNeS Ltd.; Cambridge, U.K.) sensible a los daños de las funciones ejecutivas ubicadas en los lóbulos prefrontales. La batería incluía medidas de fluencia verbal, reconocimiento espacial, patrones visuales y memoria de reconocimiento visoespacial, flexibilidad cognitiva (con una tarea similar al Wisconsin Card Sorting Test -WCST-), memoria de trabajo espacial, planificación y secuenciación. Existieron diferencias cualitativas en el funcionamiento cognitivo entre los consumidores crónicos de anfetaminas y los de heroína, especialmente en medidas de flexibilidad cognitiva, aprendizaje de discriminación visual y algunos aspectos de la función memorística visoespacial. El resultado más llamativo de este estudio fue el del deterioro específico del rendimiento en el índice EDS (equivalente al cambio de categoría del WCST) de la tarea de flexibilidad cognitiva en el grupo de consumidores de anfetaminas, déficits en este índice de la prueba habían sido previamente observados en pacientes con desórdenes asociados a los ganglios basales como la enfermedad de Huntington (Lawrence et al., 1996) y en pacientes quirúrgicos con escisiones en el lóbulo frontal permaneciendo intacto el temporal (Owen et al., 1991). En contraste, el grupo de consumidores de heroína estaba más dañado en 
estadios más tempranos del test, concretamente en el índice IDS, una medida de aprendizaje y abstracción que sugiere déficits en los procesos de aprendizaje y reforzamiento, estos sujetos mostraron también déficits selectivos en tareas sensibles a las funciones ejecutivas en dominios visuales, datos que contradicen investigaciones previas que suponían preservación de las funciones ejecutivas tras consumo prolongado de heroína (Hill y Mikhael, 1979).

Estos resultados apoyan la hipótesis de una disfunción frontal-ejecutiva en consumidores crónicos de drogas apuntada en el estudio de Rogers (Rogers et al. 1999) y son consistentes con la evidencia de que el abuso de anfetaminas está asociado a la reducción de los niveles de serotonina en el córtex orbitofrontal (Wilson et al., 1996).

La principal implicación de estos datos es que tanto el consumo de anfetaminas como el de heroína pueden provocar déficits neuropsicológicos a través de sus efectos perdurables sobre el circuito corticoestriatal, conclusión apoyada por los estudios de Volkow et al. (1997) acerca de la respuesta al metilfenidato en consumidores de cocaína o Strickland et al. (1993) sugiriendo hipoperfusión en áreas periventriculares, frontales y en otras regiones neocorticales.

En línea con estos estudios, Bechara et al. (2001), observaron que pacientes con lesiones ventromediales (VM) bilaterales y sujetos consumidores de sustancias mostraban conductas muy similares: (1) niegan o no son conscientes de su propio problema (anosognosia); (2) tienden a escoger el reforzamiento inmediato y a ignorar posibles consecuencias negativas a largo plazo. Usando una tarea computerizada (Gambling Task) de toma de decisiones en condiciones de ganancia y riesgo, diversos estudios habían mostrado déficits en consumidores de cocaína (Grant, Conttoreggi y London, 2000) y opiáceos (Petry, Bickel y Arnett, 1998). Utilizando otros instrumentos de toma de decisiones similares a la Gambling Task, diversos estudios habían mostrado asímismo que pacientes con lesiones ventromediales (VM) y consumidores de cocaína y cannabis rendían deficitariamente en estas tareas pese a que en ninguno de los grupos consumidores se ha observado una región ventromedial anormal, ni en resonancia magnética estructural ni en neuroimagen funcional (Stapleton et al., 1995; Volkow et al., 1991). Bechara et al. (2001) utilizaron la Gambling Task, junto con otra serie de tareas sensibles a las funciones ejecutivas para comparar a estos pacientes con lesiones ventromediales (VM) con consumidores crónicos de drogas.

La lógica de la tarea de la Gambling Task emula una típica situación de juego de cartas: consiste en coger más tarjetas de aquellos montones en los que se obtienen menores cantidades relativas en ganancias, pero también menores pérdidas (montones ventajo- sos) y menos de aquellos en los que las ganancias relativas pueden ser más altas, pero también mucho más altas las penalizaciones de pérdida (montones desventajosos).

Los resultados mostraron que no existían diferencias significativas entre los grupos experimentales en las principales medidas clásicas de funciones ejecutivas (WCST, Torre de Hanoi y Stroop), ambos grupos experimentales mostraron deterioro significativo en el rendimiento respecto a los controles.

En la Gambling Task, los pacientes ventromediales mostraron una tendencia a coger cada vez más cartas de los montones desventajosos, mientras que los consumidores de sustancias no se mostraron tan dañados como los VM, pero sí más que los controles. Los análisis estadísticos arrojaron diferencias significativas entre ambos grupos experimentales y el grupo control, y no hallaron diferencias significativas entre el rendimiento de los pacientes ventromediales y los consumidores de sustancias, si bien hay que tomar en consideración el hecho de que la ejecución de el grupo consumidor de drogas no fue homogénea, rindiendo algunos miembros del grupo a la altura de los controles mientras que otros rendían tan pobremente como los pacientes ventromediales. No obstante, los resultados de este estudio nos permiten sugerir la hipótesis de que un córtex ventromedial disfuncional puede estar a la base de conductas de perseveración en la búsqueda de sustancias a pesar de las consecuencias adversas asociadas.

En función de los resultados obtenidos por los diversos estudios citados, podemos sugerir la existencia de un deterioro neuropsicológico significativo en las funciones ejecutivas de sujetos consumidores de distintas drogas (cannabis, cocaína, anfetaminas y opiáceos), este deterioro podría estar implicado en (a) la asignación de una valencia emocional exagerada a las propiedades reforzantes de la droga; (b) la existencia de mecanismos disfuncionales de control de impulsos; y (c) déficits en la calidad de los procesos de toma de decisiones.

\subsection{Alteraciones del funcionamiento diario asocia- das a los deterioros neuropsicológicos.}

Son escasos los estudios que analizan las posibles implicaciones de los deterioros neuropsicológicos asociados al abuso de sustancias sobre los patrones específicos de pensamiento y el funcionamiento diario de los sujetos consumidores de drogas, pese a que: (a) la rehabilitación neuropsicológica debe orientarse a conseguir el máximo nivel de reincorporación posible del sujeto a su contexto bio-psico-social pre-mórbido; (b) en la práctica, muchos de estos deterioros comprometen seriamente la independencia y la eficiencia de los sujetos consumidores a la hora de afrontar un 
amplio espectro de situaciones cotidianas relacionadas o no con el consumo.

Bennet (1987) advirtió que estos pacientes, durante el proceso de deshabituación, sólo comprenden parcialmente lo que escuchan y observan cuando interactúan con otros, de modo que pueden generar un esquema cognitivo distorsionado que convierta a las personas que le rodean en hipercríticos, sobredemandantes e incluso conspiratorios.

Miller (1990) apreció que los sujetos consumidores tendían a ser depresivos, irritables, impulsivos, perseverativos y privados de autocontrol y autorregulación. Déficits de autorregulación y control de impulsos han sido consistentemente observados en este tipo de pacientes (Bechara et al., 2001; Rogers et al., 1999), por lo que muchos de los comportamientos socialmente inadecuados tradicionalmente asociados a los síntomas del síndrome de abstinencia, así como la tendencia compulsiva a perseverar en el consumo y la alta tasa de recaídas, que se producen incluso mucho tiempo después de haber superado el periodo de deshabituación, podrían ser explicados, al menos parcialmente, en función alteraciones neuropsicológicas específicas de las funciones prefrontales. Curiosamente, la habilidad para mantener un empleo remunerado era uno de los mejores predictores de buen rendimiento en la Gambling Task (tarea sensible a los déficits en control de impulsos y toma de decisiones), a pesar del consumo crónico de sustancias.

Un trabajo de Lundqvist (1995) compendiaba y sistematizaba una serie de observaciones clínicas realizadas durante el tratamiento de consumidores crónicos de cannabis. Estas observaciones, que conectaban los déficits en las diversas funciones cognitivas con sintomatología conductual, pensamientos y sentimientos propios de estos sujetos, sugerían que los déficits en habilidad verbal llevaban al paciente a sentirse solo e incomprendido, sus déficits en habilidad lógico-analítica le hacían sentir inadecuado e inexitoso, sus déficits en flexibilidad cognitiva le suponían dificultades para entender el punto de vista de otros, para modificar el esquema de pensamiento en la resolución de problemas cotidianos y en los procesos de percepción social y sus problemas de memoria le llevaban a exhibir falta de paciencia. Otras sensaciones registradas son las de sentirse diferente y único (asociada a déficits de habilidades analítico sintéticas), la de no pertenecer a esta sociedad o la de estar viviendo en un mundo propio (déficits de habilidad psicoespacial y memoria holística).

En opinión de estos clínicos, los sujetos tardaban hasta dos semanas en comenzar a recuperar el control de su propio funcionamiento cognitivo y en torno a seis semanas hasta mostrar un funcionamiento cognitivo normal (Tunving y Lundqvist, 1988). Siempre en base a estas observaciones, el autor sugería que el patrón específico de pensamientos observado en estos sujetos podría ser resultado de una disfunción prefrontal transitoria, dado que los lapsos de recuperación del control del funcionamiento cognitivo se solapan con los de normalización del flujo sanguíneo cerebral (Tunving et al., 1985), y que los síntomas conductuales registrados se asemejan a los que se asocian al síndrome prefrontal.

La relevancia clínica del estudio de Lunqvist pone de manifiesto la necesidad de conectar íntimamente el deterioro en determinadas funciones cognitivas con sus posibles implicaciones sobre las pautas de conducta diaria, las reacciones emocionales características y los patrones de pensamiento específicos de los sujetos consumidores de drogas con el objeto de optimizar la eficacia de las terapias (Rogers y Robins, 2001) y, como consecuencia directa, los resultados del tratamiento.

\section{4. ¿Cómo y Dónde producen las drogas deterio- ros neuropsicológicos?}

Una vez delimitado el conjunto de posibles deterioros neuropsicológicos que pueden producirse como consecuencia del consumo de sustancias, nuestro siguiente objetivo consiste en determinar (a) "¿cómo?": a través de qué mecanismos cerebrales, y (b) "¿dónde?: en qué áreas específicas producen las drogas estos deterioros. Para cumplir este doble objetivo, nos serviremos de distintos tipos de estudios que han utilizado diferentes herramientas de investigación: modelos animales, análisis de metabolitos y técnicas de neuroimagen.

El consumo de drogas puede generar deterioros neuropsicológicos a través de diversos mecanismos de acción. En primer lugar, pueden generar alteraciones morfológicas en la estructura del cerebro: pérdida de volumen cerebral, reducciones del porcentaje de materia gris, reducciones del volumen del fluido cerebroespinal ventricular, ensanchamientos del espacio pericortical y de ambos ventrículos laterales, decrementos del tamaño de las neuronas y muerte neuronal o atrofia cerebral.

Asimismo, pueden ejercer sus efectos nocivos a través de la reorganización metabólica de los circuitos de conectividad sináptica que se produce como consecuencia de los procesos de tolerancia, abstinencia y deshabituación: provocan adaptaciones bioquímicas en los sistemas de proyección de la dopamina, la serotonina y la noradrenalina, interaccionan con los receptores del glutamato y pueden bloquear los mecanismos de potenciación y depresión a largo plazo en el hipocampo y en el núcleo accumbens.

Por último, pueden provocar alteraciones en la vascularización cerebral: vasoconstricción, hemorragia cerebral paraencimal y subaracnoidea e infarto cerebral isquémico. 
Para resolver la cuestión del "¿dónde?", diversas líneas de investigación han incorporado la utilización de técnicas de neuroimagen al estudio de los deterioros neuropsicológicos asociados al consumo de drogas. Si bien el perfil de deterioro identificado es aún difuso y multifocal, las áreas que se han mostrado más consistentemente afectadas son las frontales (sobre todo el córtex orbitofrontal y sus proyecciones sobre las estructuras subcorticales que configuran el sistema de recompensa cerebral: área tegmental ventral, núcleo accumbens, núcleo mediodorsal del tálamo, giro cingulado anterior, amígdala) y las temporales (especialmente, el hipocampo).

Las alteraciones estructurales de la morfología cerebral han sido constatadas en consumidores de cannabis, cocaína, anfetaminas y opiáceos tanto de forma global como en áreas específicas prefrontales, temporales y periventriculares.

Wilson et al. (2000) vincularon el inicio temprano del consumo de marihuana (edades inferiores a 17 años) con anormalidades estructurales que incluían reducciones del volumen cerebral global y del porcentaje de materia gris en un estudio utilizando Tomografía por Emisión de Positrones (PET). Asimismo, Block et al. (2000), con Resonancia Magnética, mostraron que el consumo frecuente de marihuana en sujetos jóvenes estaba relacionado con decrementos del volumen del fluido cerebroespinal ventricular.

Liu et al. (1998) mostraron la existencia de correlaciones negativas entre la cronicidad del consumo de cocaína y el volumen prefrontal. Alteraciones en la morfología de las dentritas y de las espinas dentríticas del córtex prefrontal y del núcleo accumbens también han sido asociadas a la administración continuada de estimulantes (cocaína y anfetaminas) hasta un mes después de la interrupción del tratamiento en ratas (Robinson y Kolb, 1998). Utilizando Tomografía Axial Computerizada (TAC) y medidas lineales del fluido cerebroespinal, Pascual-Leone et al. (1991) observaron incrementos en la anchura de los ventrículos laterales y la cisura de Silvio en consumidores habituales de cocaína, mientras que Bartzokis et al. (2000), con Resonancia Magnética (MRI) describieron reducciones significativas del volumen de los lóbulos temporales tanto en consumidores de cocaína como de anfetaminas, así como una aceleración del proceso de reducción del volumen de materia gris temporal normalmente asociado a la edad. No se encontraron diferencias significativas entre consumidores y no consumidores respecto al volumen frontal.

En opiáceos, diversos estudios han detectado ensanchamiento ventricular (Strang y Gurling, 1989; Wiesbeck y Taeschner, 1991), mientras que otros no han hallado cambios morfológicos reseñables (Amass et al., 1992; Aasly et al., 1993) pese a que son muchas las claves que inducen a sugerir la existencia de alteraciones estructurales asociadas al consumo, entre ellas el decremento del tamaño neuronal asociado a la administración de morfina en ratas (Sklair-Tavlon et al., 1996) o la atrofia cerebral asociada a estados anóxicos que son frecuentes entre consumidores duros de heroína (Leeds et al., 1983). Pezawas et al. (1998), intentaron subsanar la posibilidad de que la no detección de déficits se debiera al uso insuficiente de medidas (Arndt et al., 1991) y utilizaron medidas lineales, planimétricas y estereológicas. En su estudio, detectaron un ensanchamiento significativo del espacio pericortical y de ambos ventrículos laterales, indicando pérdida de volumen cerebral en todos los grupos consumidores, si bien la pérdida de volumen frontal era significativamente mayor en aquellos sujetos que llevaban menos tiempo abstinentes respecto a los que presentaban un periodo de abstinencia superior a doce meses. La pérdida de volumen frontal estaba asociada a pobres resultados en la prevención de recaídas.

Por último, Liu et al. (1998) en un estudio de MRI, constataron reducciones significativas del volumen prefrontal en sujetos con historial de policonsumo, también se apreciaron reducciones no significativas en ambos lóbulos temporales, posiblemente como consecuencia de la gran cantidad de interconexiones entre ambas zonas.

Déficits metabólicos han sido detectados preferentemente en áreas prefrontales-subcorticales de consumidores de cannabis y estimulantes, y áreas frontales y parieto-temporales de consumidores de opiáceos.

Vokow et al. (1996) registraron alteraciones metabólicas en el córtex orbitofrontal y los ganglios de la base como consecuencia de los efectos neurotóxicos delTHC.

El grupo de Volkow (Volkow et al., 1991) había constatado ya previamente la presencia de decrementos metabólicos significativos localizados en diversas regiones frontales (incluyendo el córtex orbitofrontal y el giro cingulado anterior) de consumidores de cocaína hasta cuatro meses después del periodo inicial de desintoxicación, utilizando Tomografía por Emisión de Positrones (PET). Estudios posteriores (Volkow y Fowler, 2000) han proporcionado evidencia de anormalidades metabólicas en el striatum dorsal, el tálamo y el córtex orbitofrontal. Otros estudios (London et al., 1990; Morgan et al., 1993), también con PET, han registrado reducciones metabólicas de hasta un 14\% de reducción global de glucosa, incluyendo todas las áreas neocorticales, los ganglios basales, el tálamo, el cerebro medio y ciertas estructuras hipocampales. Vollenweider et al. (1998) detectaron alteraciones metabólicas significativas en el tálamo, el giro cingulado anterior, y los núcleos caudado y putamen utilizando PET en voluntarios sanos que habían recibido dosis controladas de anfetaminas. 
Diversos estudios efectuados con SPECT -Single Photon Emisión Computed Tomographic- (Levin et al., 1994; Strickland y Stein, 1995) muestran asímismo hipoperfusión en áreas cerebrales multifocales que en algunos sujetos persisten desde meses hasta años después de haber interrumpido el consumo, la hipoperfusión periventricular ha sido observada en consumidores de cocaína tanto como consecuencia de los efectos agudos de la droga (Pearlson et al., 1993), como después de un abuso crónico (Strickland et al., 1993). Strickland et al. (1998) correlacionaron la propensión a presentar déficits de memoria, atención, funcionamiento ejecutivo y velocidad de procesamiento mental observados en consumidores crónicos de cocaína con alteraciones metabólicas y de flujo cerebral registradas especialmente en áreas frontales subcorticales.

En opiáceos, Rose et al. (1996) detectaron déficits de perfusión, especialmente en las cortezas frontales, temporales y parietales de consumidores crónicos de heroína después de una semana de abstinencia, en este estudio, las anormalidades del flujo sanguíneo cerebral no se correspondían con alteraciones estructurales ni morfológicas de la anatomía cerebral, al mostrarse estas alteraciones en la perfusión parcialmente reversibles tras una relativamente corta abstinencia. Gerra et al. (2000), utilizando SPECT, hallaron decrementos no significativos del flujo sanguíneo cerebral en áreas frontales, parietales y en la región temporal izquierda de la corteza de pacientes crónicos dependientes de opiáceos tras cuatro meses de abstinencia.

Asimismo, hay que tener en cuenta la capacidad de la cocaína y otros estimulantes para provocar indirectamente daños neuropsicológicos a través de su acción sobre otros órganos que influencian la fisiología del cerebro (Kosten, 1997; Strickland et al., 1998), el abuso de cocaína ha sido asociado a hemorragias cerebrales paraencimales en el córtex cerebral, en los ganglios de la base y en el tronco cerebral asociadas a hipertensión severa (Grewal y Miller, 1991) así como a infartos cerebrales isquémicos (Mody et al., 1998) sin estar necesariamente presentes, en este último caso, alteraciones cerebrovasculares previas.

En conclusión, y en función de la literatura acumulada, podemos concluir: (a) que diversas drogas de consumo provocan alteraciones estructurales y metabólicas significativas en áreas corticales y subcorticales de crucial importancia para el funcionamiento neuropsicológico del sujeto, y (b) que estas alteraciones cerebrales correlacionan con el perfil de deterioro neuropsicológico característico de los sujetos drogodependientes, aunque (c) serían necesarios más estudios que pusieran en relación la existencia de alteraciones cerebrales subyacentes con la presencia de déficits neuropsicológicos concretos.

\section{RELEVANCIA E IMPACTO DE LAS ALTERACIO- NES NEUROPSICOLÓGICAS EN LA PRÁCTICA CLÍNICA CON DROGODEPENDIENTES.}

Una vez establecidos los principales deterioros neuropsicológicos que pueden producirse como consecuencia del consumo crónico de drogas, así como las áreas y mecanismos cerebrales implicados en su aparición, analizaremos las principales implicaciones que se derivan de la existencia de estos deterioros para la práctica clínica con drogodependientes. La aparente reversibilidad de los déficits (tanto en poblaciones adultas como adolescentes), la co-existencia de trastornos de la personalidad y del estado de ánimo, y la presencia de anosognosia y déficits específicos de control de impulsos y toma de decisiones son factores que pueden influenciar considerablemente las condiciones, estrategias, herramientas y parámetros temporales del tratamiento, así como sus resultados y potencial eficacia. Resulta conveniente, por tanto, analizar la relevancia e impacto de estos factores asociados a alteraciones neuropsicológicas sobre la práctica clínica con sujetos consumidores.

\subsection{Reversibilidad de los déficits neuropsicológi- cos asociados al consumo de drogas: implica- ciones para el tratamiento y la rehabilitación.}

Entre los posibles déficits neuropsicológicos que pueden resultar del consumo prolongado de sustancias estarían: (a) los que son propios de la intoxicación inducida por la droga; (b) los que se producen como consecuencia de los efectos agudos de la sustancia y (c) aquellos que persisten una vez que la droga ha abandonado el organismo, como consecuencia de alteraciones perdurables en el funcionamiento del Sistema Nervioso Central (Pope Jr. et al., 1994).

Allen y Landis (1998) sistematizaron los hallazgos neuropsicológicos que tradicionalmente se habían asociado al consumo de drogas en función del tipo de sustancia y de estas tres categorías. Los consumidores de cannabis mostraban déficits en resolución de problemas, abstracción, atención, lenguaje expresivo, receptivo y memoria como consecuencia de la intoxicación inducida por el THC (delta-9-tetrahydrocannabidol), los deterioros resultantes de los efectos agudos de la droga (hasta 24 horas después de la intoxicación) abarcaban tareas de atención, funciones ejecutivas, aritmética mental y recuperación inmediata de información verbal. En cambio, a largo plazo, aquellos estudios que han logrado constatar la existencia de efectos perdurables del cannabis sobre el SNC (Mendhiratta et al., 1988; Schwartz et al., 1999) ubican los déficits en atención, memoria a corto plazo y funciones viso-motoras. 
Asimismo, en opiáceos, mientras que los efectos agudos de la droga inducen deterioros en memoria visual y verbal demorada, atención y concentración, fluencia verbal y habilidades viso-espaciales y visomotoras, los déficits a largo plazo incluyen funciones ejecutivas y razonamiento no verbal.

Por otro lado, en consumidores de cocaína, el espectro de funciones dañadas también se reduce respecto a los estudios que detectan efectos agudos (Beatty et al., 1995; Berry et al., 1993)- cuando analizamos sus efectos residuales (Strickland et al., 1993; Selby y Azrin, 1997).

Existen datos suficientes para sugerir que los déficits cognitivos detectados en sujetos consumidores de sustancias no son estáticos, sino que experimentan mejoras dinámicas una vez que se inicia la abstinencia. Hay una cantidad significativa de recuperación de funciones neuropsicológicas durante el primer mes de abstinencia, especialmente en áreas de aprendizaje verbal, procesamiento viso-espacial y destrezas motoras (Goldman, 1983; Goldman, Williams y Klisz, 1983; Parsons y Leber, 1982). La memoria a corto plazo y la velocidad motora continúan mejorando durante los meses y años subsiguientes (Klisz y Parsons, 1977; Schau, O'Leary y Chaney, 1980). En cambio, la recuperación de ciertas destrezas como el razonamiento abstracto o la resolución de problemas puede llevar mucho más tiempo y en muchos casos no retornar nunca a niveles normales (Effenberg et al., 1980; Goldman, 1983). Jasiukaitis y Fein (1999), resumiendo las conclusiones de diversos estudios constataron la existencia de recuperación moderada de funciones entre la quinta y sexta semana y el sexto mes de abstinencia en consumidores de cocaína. Gillen et al. (1998) correlacionaron negativamente la cantidad de daño con el periodo de abstinencia transcurrido. La duración de la abstinencia ha sido positivamente correlacionada con la mejora del rendimiento cognitivo en consumidores de alcohol, cocaína y en policonsumidores (Azrin et al., 1992; Yuspeh et al., 1992). Selby y Azrin (1997) demostraron mejoras del funcionamiento neuropsicológico en un $60 \%$ de las medidas para consumidores de alcohol y de un $33 \%$ para policonsumidores.

Existe, por tanto, reversibilidad o recuperación de los déficits neuropsicológicos asociados al consumo de drogas. Esta reversibilidad parece estar relacionada con la duración de la abstinencia y ocurre en grado diferencial para las distintas funciones afectadas, de modo que algunas de ellas recuperan rápidamente, mientras que otras -caso de las funciones ejecutivasrecuperan más lentamente y en raras ocasiones logran retornar a sus niveles premórbidos. Alcanzado este punto, y desde una perspectiva clínica, se nos plantea una segunda cuestión de crucial relevancia. Dado que existe reversibilidad de los déficits, ¿resulta conveniente aplicar estrategias de rehabilitación neuropsicológica sobre las funciones dañadas?

Strickland et al. (1993) advierten que aunque la recuperación ocurre de hecho durante la abstinencia inicial, déficits cognitivos y anormalidades neuropsicológicas persistentes son hallados a menudo, incluso después de prolongados periodos de abstinencia. Estos déficits específicos podrían resultar de especial relevancia para el éxito de las actuales estrategias de intervención terapéutica en drogodependencias.

Durante la fase de deshabituación, en la que se establecen la filosofía, las normas y los objetivos del programa terapéutico y se implementan diversas técnicas de importante carga cognitiva: reestructuración de pensamientos, terapia racional emotiva, deterioros específicos en atención, concentración y memoria a corto plazo pueden comprometer la asimilación de diversos contenidos relevantes del programa. Mantener la atención durante prolongadas sesiones terapéuticas puede resultar -por ejemplo- extraordinariamente dificultoso para los sujetos consumidores debido a la pervivencia de estos déficits. Asimismo, déficits de memoria a corto plazo y de memoria prospectiva (recordar tener que recordar) pueden dificultar la consolidación de material clave referente al tratamiento y la ejecución de los ejercicios y tareas que los terapeutas les sugieren. Para Rogers y Robbins (2001), los daños neuropsicológicos pueden contribuir al abuso y la adicción a las drogas de al menos dos formas. En primer lugar, pueden interferir la capacidad de los usuarios para asimilar y participar en programas de rehabilitación que tengan énfasis educativo o cognitivo, y en segundo lugar, pueden incrementar la probabilidad de conductas de búsqueda de sustancias a través de distintos tipos de déficits cognitivos, incluyendo fracasos en los mecanismos de control de impulsos.

Los deterioros neuropsicológicos detectados en las funciones ejecutivas prefrontales del córtex ventromedial y sus conexiones con diversas áreas funcionales subcorticales (amígdala, núcleo accumbens) suponen un problema añadido para el objetivo de implementar estrategias de mantenimiento de la abstinencia y resistencia a los impulsos de consumo, especialmente durante la fase de prevención de recaídas. Estos deterioros pueden afectar: (1) a la capacidad de los sujetos consumidores para aprender de la experiencia, debido a su incapacidad para re-experimentar y evocar estados emocionales asociados al castigo (Bechara, et al., 2000); (2) a su habilidad para prevenir recaídas, ya que en sus procesos de toma de decisiones otorgan una valencia emocional exagerada a los estímulos potencialmente reforzantes asociados a la droga (Di Chiara, 1999), presentan déficits de control de impulsos (Volkow y Fowler, 2000) y exhiben una ausencia de respuesta emocional previa a la elección de consecuencias desventajosas (Bechara et al., 
2000); (3) a su habilidad para buscar nuevas fuentes de recompensa, ya que tienen muchísimos problemas para identificar objetivos y metas relevantes y para organizar y monitorizar su propia conducta en función de esas metas (Mateer, 1997); (4) y en conjunto, a sus habilidades para intentar cambiar de estilo de vida, en función de sus problemas de iniciación, secuenciación y planificación de conducta y de posibles alteraciones de la personalidad (Prigatano, 2001).

Por tanto, y aunque podemos asumir la existencia de cierta recuperación espontánea asociada a la abstinencia, pueden permanecer dañadas funciones específicamente relevantes para el éxito en la manutención de esa abstinencia y la prevención de posibles recaídas. Fals-Stewart et al. (1992; 1993; 1994a; 1994b) sugirieron que la presencia de déficits neuropsicológicos estaba relacionada con incrementos de la violación de reglas, peores estimaciones clínicas y mayores tasas de abandono involuntario de tratamientos de desintoxicación y deshabituación. Dado el curso natural de recuperación de las funciones neuropsicológicas en este tipo de población y el auge de los programas de tratamiento de la adicción a muy corto plazo, muchos de estos sujetos no alcanzan la recuperación total de funciones durante el transcurso del tratamiento, y suelen concluirlo justo en el momento en que sus habilidades cognitivas han experimentado una recuperación suficiente como para obtener efectos beneficiosos del tratamiento, además de regresar a su entorno en condiciones de alto riesgo respecto a la ocurrencia de recaídas. Éste es uno de los retos más importantes a los que debe enfrentarse el neuropsicólogo en la práctica clínica con sujetos consumidores de drogas, puesto que en función de los parámetros temporales impuestos por la estructura externa del programa de tratamiento, debe ajustar la rehabilitación del paciente al ritmo del programa en unas condiciones neuropsicológicas que distan de ser óptimas, y tratar de reincorporarlo a su contexto de origen asumiendo el menor número de riesgos posible.

No obstante, Sohlberg y Mateer (1989), que realizaron una revisión exhaustiva de los procedimientos de rehabilitación cognitiva en sujetos consumidores constataron que tanto el número de funciones recuperadas como el grado de recuperación incrementaban como consecuencia de la aplicación de estrategias de rehabilitación cognitiva. El entrenamiento cognitivo parece acelerar la reversibilidad de los déficits e incluso optimizar la recuperación de funciones por encima de los niveles que podrían ser alcanzados mediante recuperación espontánea, y dado que el estatus neuropsicológico parece tener un papel mediador sobre los resultados de tratamiento, este incremento en la recuperación puede resultar clave para el éxito del mismo.

Aunque aún no disponemos de un protocolo establecido de rehabilitación de estas funciones neuropsi- cológicas que haya mostrado su eficacia diferencial respecto a los programas de intervención que actualmente se utilizan en este tipo de población, la constatación de la existencia de un perfil bien delimitado de deterioro, la eficacia demostrada por las estrategias rehabilitación neuropsicológica en otras poblaciones con déficits similares, y los nuevos hallazgos y posibles focos de intervención revelados por los estudios más novedosos en el campo (autorregulación, control de impulsos, toma de decisiones), permiten hipotetizar los beneficios potenciales de un abordaje neuropsicológico de estos déficits integrado en el marco de un programa de intervención que incluya otras estrategias de intervención psicológicas y farmacológicas.

A partir de los hallazgos neuropsicológicos más recientes en relación al abuso de sustancias y de sus principales implicaciones clínicas, podemos sugerir que estas estrategias de rehabilitación neuropsicológica deben focalizarse en los siguientes objetivos: (a) optimizar los mecanismos de procesamiento de información, atención, concentración y memoria, funciones que se han mostrado consistentemente dañadas como consecuencia del consumo (Ardila y Roselli, 1996; Strickland et al., 1998) y cuya recuperación propiciaría una mejor asimilación de las normas, filosofía y contenidos de los programas de tratamiento (Goldman, 1990); (b) incidir en la recuperación de las funciones ejecutivas localizadas en los lóbulos prefrontales: flexibilidad cognitiva, resolución de problemas, mecanismos de control de impulsos y toma de decisiones (Bechara et al., 2001; Ornstein et al., 2000; Rogers et al., 1999; Volkow y Fowler, 2001) que permitirán al sujeto: mejorar su funcionamiento diario, mejorar su resistencia a posibles estresores (Estímulos Condicionados asociados a la droga), incrementar su capacidad de autocontrol, prevenir conductas de búsqueda y regresar a su entorno en condiciones óptimas para afrontar decisiones relacionadas con el consumo-no consumo.

\subsection{Peculiaridades de la población adolescente en la reversibilidad y recuperación de los déficits neuropsicológicos.}

La población adolescente ha sido frecuentemente ignorada en estudios referentes al deterioro neuropsicológico asociado al consumo de sustancias, pese a que la prevalencia del consumo de drogas ha incrementado sensiblemente en adolescentes y jóvenes adultos durante las últimas décadas (Hoover, 1991), y a pesar de sus implicaciones negativas directas sobre diversos aspectos sociales y educativos (Newcomb y Bentler, 1988). Los resultados obtenidos en estudios realizados con adultos no pueden ser directamente trasladados a esta población (Riggs et al., 2000), puesto que durante la adolescencia se producen importan- 
tes desarrollos neurológicos que pueden influenciar los efectos de las drogas sobre el funcionamiento neuropsicológico de estos individuos (Tapert y Brown, 1998), tanto respecto a la magnitud del deterioro como a la cantidad de recuperación alcanzable. Las áreas de asociación frontales y parietales, que aparecen consistentemente asociadas al deterioro provocado por el consumo de drogas, son las últimas en mielinizarse (Kolb y Fantie, 1989), lo que sugiere que la velocidad de transferencia de información en esas regiones es relativamente insuficiente en torno a la edad de 15 años, las conexiones sinápticas desaparecen en función de la redundancia y la estimulación ambiental selectiva entre los 7 y los 16 años (Huttenlocher, 1990), los hemisferios cerebrales continúan su lateralización (Rourke et al., 1983), con maduración de la materia gris global, de la materia blanca frontal y de regiones talámicas (Steen et al., 1997).

Estos desarrollos tienen importantes implicaciones para la influencia neurotóxica de las drogas, posiblemente permitiendo una mayor elasticidad y resistencia respecto a los adultos (Tapert y Brown, 1998). No obstante, alternativamente, la exposición neurotóxica durante un momento crucial del desarrollo neurológico puede interrumpir o impedir la maduración neuropsicológica (Rourke et al., 1983). La existencia de una mayor plasticidad o resistencia a los conocidos efectos dañinos de las sustancias adictivas sobre estructuras y funciones cerebrales, así como la posibilidad de que la inmadurez en el desarrollo pudiera implicar una mayor vulnerabilidad a estos efectos (plasticidad frente a periodicidad) son fenómenos neurológicos específicos de esta etapa y deben ser consideradas en la práctica clínica, puesto que convierten la rehabilitación neuropsicológica en una herramienta clave para afrontar la recuperación de potenciales deterioros durante este periodo concreto.

Otra cuestión sin dilucidar y que resulta de especial relevancia en población adolescente es la de la dirección causa-efecto, diversos estudios sugieren la posibilidad de que los deterioros neuropsicológicos detectados en adolescentes consumidores de drogas pueden ser debidos a la co-existencia de trastornos del desarrollo y de la personalidad. Trastornos de inatención, impulsividad e hiperactividad (Barkley et al., 1990; Biederman et al., 1996), así como agresividad infantil y desarrollo de conductas antisociales (Brook et al., 1995), la existencia de progenitores consumidores (Tarter et al., 1989; Moss et al., 1992) y de trastorno depresivo mayor (Pogge et al., 1996) pueden estar a la base de patologías neurológicas leves (Roselli y Ardila, 1996) que precipiten el consumo de sustancias y contribuyan a la magnitud del deterioro provocado por éste. Parece probada la existencia de un rasgo de disregulación conductual -que incluye inatención, impulsividad, hiperactividad y agresividad (Dawes, Tarter y Kirisci, 1997; Giancola et al., 1996; Henry et al.,
1996)- que sugiere la existencia de un desfase en el proceso de maduración frontal (Pontius y Ruttiger, 1976) y que discrimina entre adolescentes consumidores y no consumidores. Otros estudios, en cambio, señalan la severidad del consumo como principal marcador del daño cognitivo (McCaffrey, 1989; Spencer, 1991). Entre ellos, Tarter et al. (1995) correlacionaron la severidad del abuso de sustancias con la magnitud del deterioro en tareas de funcionamiento verbal, atención y eficiencia perceptiva. Estos autores sugirieron la existencia de un rasgo de impulsividad entre los sujetos consumidores que podría estar mediado en parte por su incapacidad para usar el lenguaje de forma eficiente como estrategia de regulación cognitiva de la conducta. En ausencia de capacidad de resolución de problemas mediada verbalmente, estos sujetos tienden a consumir para proporcionarse un alivio inmediato en situaciones estresantes. La función lingüística debe ser, por tanto, uno de los objetivos clínicos de la rehabilitación de jóvenes consumidores.

Tapert y Brown (1998), en un estudio de 4 años de seguimiento del estatus neuropsicológico de adolescentes consumidores hallaron que la severidad de las experiencias de retirada parecía estar particularmente asociada a la magnitud del daño cognitivo en esta población. Los adolescentes que auto-informaban de mayores síntomas de retirada puntuaban por debajo de otros jóvenes consumidores en tareas de procesamiento no verbal, los informes acerca de alguna experiencia de retirada durante los últimos tres meses correlacionaban negativamente con el rendimiento en tareas viso-espaciales, atencionales y de resistencia a la intrusión. Glenn et al. (1988) había sugerido previamente la relación entre procesos de retirada y déficits memorísticos. Ambos estudios sugieren la posibilidad de que las secuelas fisiológicas del consumo de drogas (los síndromes de retirada) pueden ser un marcador de riesgo de daño neuropsicológico más potente en consumidores jóvenes que en adultos. Posiblemente la mayor plasticidad del sistema nervioso central en los adolescentes les permite mantener un funcionamiento neuropsicológico consistente a pesar de la exposición a neurotoxinas, pero sólo hasta que los niveles de consumo inducen dependencia física.

Por último, otro rasgo característico del consumo en adolescentes que puede influenciar la magnitud del daño y la capacidad de recuperación es la alta prevalencia de consumo de inhalantes, al haberse mostrado estos como una de las sustancias potencialmente más dañinas para el funcionamiento neuropsicológico de consumidores jóvenes, incluyendo deterioros en funciones atencionales, memorísticas, viso-espaciales y motoras finas (Allison y Jerrom, 1984; Berry et al., 1978; Bigler, 1979).

En base a la evidencia acumulada por los aún escasos estudios que hacen referencia a los deterioros neuropsicológicos asociados al consumo de drogas 
en adolescentes, desde una perspectiva clínica recomendamos (a) tomar en consideración la existencia de una mayor plasticidad sináptica que puede contrarrestar los daños que la exposición a neurotoxinas pueden provocar en un cerebro aún en desarrollo mediante el uso de estrategias rehabilitadoras apropiadas; (b) poner especial énfasis en la recuperación de funciones lingüísticas y atencionales, por su crucial relevancia en la regulación de mecanismos de control de impulsos que parecen estar a la base de la conducta de consumo; (c) tener en cuenta la posible co-existencia de trastornos del desarrollo y de la personalidad y la necesidad de tratar su sintomatología de forma monitorizada y específica y (d) controlar aquellos factores (tipo de drogas, severidad de los síntomas de retirada) que se han mostrado potencialmente responsables de un incremento de la cantidad de deterioro neuropsicológico y que pueden condicionar las posibilidades de recuperación.

\subsection{Comorbilidad con Trastornos del Estado de Ánimo.}

Diversas investigaciones proponen que la vulnerabilidad a la depresión o a la distimia constituye una de las principales motivaciones para el consumo de sustancias (Gawin y Cléber, 1986; Weiss, Griffin y Mirin, 1992). Esta hipótesis es consistente con el hecho de que sujetos consumidores de drogas presenten una alta prevalencia de trastornos del estado de ánimo (Rounsaville et al., 1991). Es plausible que los efectos dopaminérgicos directos de las drogas estimulantes sobre el cerebro puedan inducir una acción antidepresiva que convierta la auto-administración de sustancias en especialmente reforzante para individuos que son vulnerables a la depresión (Gunnarsdottir et al., 2000). Roselli y Ardila (1996) detectaron que grupos consumidores de cocaína y policonsumidores exhibían diferencias significativas respecto a sujetos controles en diversas áreas de desarrollo que incluían auto-agresión, ansiedad excesiva e ideaciones e intentos de suicidio, lo que sugería la presencia de rasgos de personalidad depresivos y ansiosos. Ansiedad o depresión junto con personalidad borderline habían sido asociados con consumo crónico y dependencia de drogas por la APA (American Psychiatric Association, 1987). Ardila y Bateman (1995) también habían registrado una asociación significativa entre dependencia de drogas y depresión.

Los Trastornos del Estado de Ánimo, además de actuar como posibles factores antecedentes del inicio del consumo, pueden también producirse como consecuencia del abuso. La cocaína produce sus efectos euforizantes mediante la inhibición de la recaptación presináptica de catecolaminas en el sistema de proyección dopaminérgico (Post, 1975; Galloway, 1988) y en los ganglios de la base (Volkow et al., 1988), sin embargo, estos receptores dopaminérgicos pueden quedar mermados (Volkow et al., 1990), convirtiéndose así en el principal factor inductor de la depresión que usualmente sigue al consumo crónico.

Strickland et al. (1998) advierten que esta debe ser una importante variable de control en la aplicación de técnicas de neuroimagen al estudio de los correlatos neuropsicológicos del abuso de drogas, puesto que las anormalidades registradas en consumidores depresivos tienden a atribuirse al efecto de las drogas, y no al de la depresión (Baxter et al., 1988).

Gerra et al. (1998), en un estudio realizado con SPECT en consumidores de heroína abstinentes durante cuatro meses, detectaron que las alteraciones de la perfusión cerebral estaban más relacionadas con variables de personalidad y trastornos del estado de ánimo que con la historia adictiva. Estos autores registraron hipoperfusión cerebral significativa en las regiones frontal derecha y temporal izquierda de sujetos adictos con depresión comórbida y detectaron la existencia de correlación negativa entre el flujo sanguíneo cerebral del área temporal izquierda y las puntuaciones en la subescala de depresión del Minessota Multiphasic Personality Inventory (MMPI).

Diversos estudios previos habían registrado correlaciones entre síntomas depresivos y anormalidades metabólicas: incremento de la perfusión en el giro cingulado y otras áreas paralímbicas y decremento en la región frontal (Mozley et al., 1996; Ebmeier et al., 1997), la existencia de una disfunción temporal dependiente de estado en el trastorno bipolar ha sido sugerida por un estudio de SPECT (Gyulai et al., 1997). Bremner et al. (1997) con PET detectaron fuertes correlaciones entre el metabolismo del córtex frontal, funcionamiento serotoninérgico y síntomas depresivos. Gunnarsdottir et al. (2000) hallaron altos índices de depresión y ansiedad entre sujetos consumidores de cocaína que presentaron hipoperfusión frontal en SPECT. Beatty et al. (1995) mostraron que tanto alcohólicos como consumidores de cocaína mostraban puntuaciones elevadas en el Beck Depresión Inventory (BDI), asímismo, las puntuaciones del BDI estaban negativamente correlacionadas con el rendimiento de los sujetos consumidores en el WCST. Sin embargo, estos autores descartaron que el rendimiento neuropsicológico estuviese consistentemente relacionado con las puntuaciones en depresión. Horner, Harvey y Denier (1999) mostraron que las autoevaluaciones de sujetos consumidores acerca de sus posibles daños cognitivos estaban fuertemente relacionadas con depresión y vulnerabilidad al estrés.

Estos trastornos comórbidos del estado de ánimo (Ansiedad, Depresión y Trastorno Bipolar) han mostrado una buena respuesta a la terapia farmacológica, en sujetos drogodependientes, debido posiblemente a la acción directa que ejercen los fármacos sobre neuro- 
transmisores implicados en el proceso adictivo (dopamina y serotonina), por lo que diversos estudios aconsejan su prescripción, cuidadosamente controlada, en pacientes que presentan este tipo de patología dual (Brown et al., 2001; Gunnarsdottir et al., 2000; Scott, Gilvarry y Farell, 1998).

Desde una perspectiva clínica, y en base a la evidencia acumulada en torno a la relación entre los trastornos del estado de ánimo y el consumo de drogas y de sus respectivos efectos sobre el estatus neuropsicológico de los pacientes, parece conveniente sugerir: (a) una evaluación exhaustiva y sensible a la presencia de este tipo de trastornos entre los sujetos consumidores; (b) la aplicación prioritaria de aquellas estrategias de rehabilitación neuropsicológica que puedan contribuir al tratamiento de estos trastornos co-mórbidos; y (c) la incorporación de terapias específicamente apropiadas para estos trastornos como instrumento integrado dentro de las estrategias de rehabilitación neuropsicológica.

\subsection{Rasgos y Trastornos de la Personalidad.}

La tasa de prevalencia de trastornos de personalidad en sujetos consumidores de sustancias es aproximadamente cuatro veces más alta que en sujetos normales (Zimmerman y Coryell, 1989). Estos trastornos no sólo se producen como consecuencia de los efectos agudos o residuales de la droga (Skodol et al., 1999), sino que pueden preceder al consumo e interactuar con el proceso adictivo en base a un modelo de diatesis-estrés.

La evidencia disponible acerca del importante papel de las patologías de la personalidad en la etiología de los trastornos por abuso de sustancias puede derivarse de (a) estudios que registran la alta co-morbilidad relativa entre ciertos trastornos de la personalidad -particularmente antisocial, borderline, paranoide y de evitación- y el consumo de drogas; (b) estudios longitudinales a largo plazo que muestran como factores de personalidad predicen el posterior inicio del consumo; y (c) estudios retrospectivos que muestran que la psicopatología resulta en numerosos casos predictiva de la aparición de problemas adictivos (Verheul, 2001).

Los factores de personalidad contribuyen al inicio del consumo a través de tres posibles rutas (Verheul y Brink, 2000): (a) ruta de desinhibición conductual, que predice que individuos que puntúan alto en escalas de antisociabilidad e impulsividad y bajo en escalas de evitación de dolor tienen umbrales inferiores para el desarrollo de conductas de consumo, especialmente de estimulantes (cocaína y anfetaminas), se correspondería con el trastorno antisocial de la personalidad y estaría relacionada con déficits serotinérgicos; (b) ruta de reducción del estrés, en sujetos que puntúan alto en escalas de reactividad al estrés, sensibilidad a la ansiedad y neuroticismo, son más vulnerables a los acontecimientos estresantes diarios y tienden a responder al estrés con ansiedad e inestabilidad emocional que puede convertirse en un motivo para el consumo como forma de auto-medicación (alcohol, heroína, benzodiacepinas), se correspondería con los trastornos de personalidad dependiente, borderline, esquizotípica y de evitación y estaría asociada al neurotransmisor GABA; (c) ruta de sensibilidad al refuerzo, predice que los sujetos que puntúan alto en escalas de búsqueda de novedad, búsqueda de recompensa, extraversión y gregarismo se verán motivados hacia el consumo de una gran variedad de sustancias en base a sus intrínsecas propiedades reforzantes. Relacionada con el neurotransmisor dopamina, hay que tener en cuenta que parte del proceso de sensibilización está inducido por la hiper-responsividad del sistema dopaminérgico mesolímbico durante el consumo, aunque lo que propone la hipótesis es que algunos individuos, caracterizados por una mayor sensibilidad general a los reforzamientos positivos, puedan desarrollar esta hiper-responsividad de forma más rápida y potente (Cloninger, 1997; Zuckerman, 1999), correspondería con trastornos de personalidad histriónica y narcisista.

Existe controversia en torno al papel que desempeñan los trastornos de la personalidad en la respuesta al tratamiento del abuso de sustancias. Diversos estudios parecían indicar que la co-existencia con trastornos del Eje 2 predecía peores resultados de tratamiento (Leal et al., 1994; Reich y Vasile, 1993; Strand y Benjamín, 1997), pero en los últimos años otra serie de estudios han mostrado que la existencia de patologías de la personalidad no parece ser un predictor fiable de la cantidad de recuperación posible (Alterman et al., 1998; Cacciola et al., 1996; Crits-Cristoph et al., 1999; Verheul et al., 1999) -con lo cual pueden beneficiarse del tratamiento en el mismo grado que aquellos que no presentan co-morbilidad (Krueger et al., 1996; Marlowe et al., 1997)-, pero sí de la severidad del consumo y, por tanto, de la cantidad de daño neuropsicológico.

Kuruoglu et al. (1996) mostraron que sujetos alcohólicos con personalidad antisocial exhibían una hipoperfusión frontal más marcada que aquellos alcohólicos que no presentaban co-morbilidad. Gerra et al., (1998) detectaron hipoperfusión significativa en el lóbulo frontal derecho de adictos a opiáceos con tendencias antisociales, estos autores sugirieron que los patrones anómalos de flujo cerebral podían predecir el inicio de la conducta de consumo y estaban relacionados con trastornos psiquiátricos co-mórbidos. Bauer (2001) demostró que sujetos dependientes de la cocaína con trastorno de personalidad antisocial ejecutaban -respecto a consumidores sin doble diagnóstico- respuestas de forma más prematura durante una tarea de estimación temporal, asimismo, el electroen- 
cefalograma del grupo con trastorno de la personalidad antisocial se caracterizaba por la emergencia de una negatividad dominante en regiones frontales cuya latencia coincidía con el periodo de tiempo que contenía este excesivo número de respuestas. La ausencia de correlación entre la habilidad de estimación temporal y la severidad del consumo de cocaína y la duración del periodo de abstinencia inducen a pensar que los daños están relacionados con factores de personalidad premórbidos. Esta activación temporalmente inapropiada de las neuronas implicadas en la preparación y ejecución de respuestas motoras (Rockstroh et al., 1982) puede estar a la base de los déficits detectados en paradigmas experimentales de toma de decisiones (Bauer, 2001). Anormalidades neuropsicológicas y de amplitud del potencial P300 focalizadas frontalmente habían sido ya previamente detectadas en consumidores de cocaína (Bauer, 1997a, b) y alcohólicos que presentaban trastorno de la personalidad antisocial, decrementos similares asociados al trastorno han sido observados en niños (Bauer y Hesselbrock, 1999) y jóvenes adultos (Bauer et al., 1994a, b; O’Connor et al., 1994) que no eran consumidores de drogas ni alcohol. Otros estudios, en cambio, no detectan diferencias significativas en deterioro neuropsicológico debidas a trastornos de la personalidad pre o co-mórbidos (Beatty et al., 1995; Easton y Bauer, 1996).

Otros autores (McGuire, 2000) consideran el consumo como principal precipitante del desarrollo de síntomas psiquiátricos crónicos que incluyen psicosis (Creighton, Black y Hyde, 1991; McCann y Ricaurte, 1991; McGuire y Fahy, 1991; Schifano, 1991), trastornos de pánico (Pallanti y Mazzi, 1992, McGuire et al., 1994a;b) y síntomas obsesivo-compulsivos (Cassidy y Ballard, 1994). En el caso de la esquizofrenia, existen hasta siete posibles hipótesis explicativas del doble diagnóstico (Tracy, Josianssen y Ballack, 1995), incluyendo tanto modelos de vulnerabilidad compartida como de sensibilidad e influencia en ambas direcciones. Pacientes esquizofrénicos y consumidores de drogas comparten un amplio espectro de déficits neuropsicológicos (abstracción, atención selectiva, procesamiento controlado, memoria episódica) y alteraciones estructurales (ensanchamiento de los ventrículos, hipometabolismo frontal, atrofia cortical) comunes. Serper et al. (2000) no consiguieron detectar diferencias significativas entre el estatus neurocognitivo de pacientes esquizofrénicos consumidores y no consumidores de cocaína. Otros autores (Goldberg et al., 1991) han sugerido incluso la posibilidad de que los efectos agudos de los psicoestimulantes puedan mejorar la sintomatología y el funcionamiento neurocognitivo de pacientes esquizofrénicos a través de la activación de las áreas corticales prefrontales. Por otra parte, el tratamiento farmacológico con antipsicóticos atípicos de nueva generación ha demostrado importantes beneficios en el tratamiento de pacientes esquizofrénicos consumidores de drogas (Littrell et al., 2001).

No está resuelta aún, por tanto, la cuestión de la influencia relativa de los trastornos de la personalidad sobre el deterioro neuropsicológico detectado en consumidores de drogas, mientras que algunos estudios sugieren la posibilidad de que la existencia de trastornos co-mórbidos puede incrementar la magnitud del deterioro (Bauer, 2001) o incluso ser la causa principal del mismo (Gerra, 1999), y otros no encuentran diferencias en el rendimiento de sujetos consumidores que sean atribuibles a la co-existencia de otros trastornos del eje 2 (Beatty et al., 1995; Easton y Bauer, 1996), estudios recientes sostienen la hipótesis de que el deterioro neuropsicológico es en sí mismo el principal causante de la aparición de diversos trastornos de la personalidad (McGuire, 2000; Prigatano, 2001).

Tampoco queda resuelta la cuestión referente a la influencia de la co-morbilidad sobre los efectos y resultados del tratamiento, por lo que desde una perspectiva clínica, este tipo de casos deben ser afrontados de una forma extremadamente prudente: (a) tratando de establecer con exactitud la naturaleza del trastorno co-mórbido y su posible influencia etiológica sobre el inicio del consumo; (b) delimitando cuidadosamente la magnitud y severidad del déficit neuropsicológico, que puede ser superior al de otros pacientes que presenten niveles similares de cronicidad y severidad en el consumo y (c) incorporando selectivamente, durante el periodo de rehabilitación, aquellas estrategias que resulten más apropiadas en función del tipo de trastorno.

\subsection{Anosognosia y problemas de control de impul- sos.}

Bechara et al. (2001) sostienen que los sujetos consumidores de sustancias, al igual que los pacientes con lesiones frontales ventromediales, se caracterizan en términos conductuales porque: (a) a menudo niegan, o no son conscientes de que tienen un problema y (b) cuando afrontan la elección de un curso de acción que les proporciona una recompensa inmediata, aun a riesgo de incurrir en futuras consecuencias negativas que incluyen la pérdida de reputación, trabajo, hogar o familiares, siempre eligen la recompensa inmediata e ignoran las consecuencias futuras.

La no conciencia del déficit (anosognosia) ha sido descrita en numerosos síndromes neuropsicológicos, neurológicos y psicopatológicos de perfil frontal como la heminegligencia atencional o la enfermedad de Alzheimer, y es de crucial relevancia para la práctica clínica, ya que los pacientes que carecen de conciencia acerca de sus propios déficits pueden sufrir falta de motivación hacia el tratamiento, fracasar en la utilización de estrategias compensatorias recomendadas, 
establecer metas no realistas o experimentar problemas de adaptación social (McGlynn y Schacter, 1989; Wagner y Cusham, 1994; Campodonico y McGlynn, 1995). Pese a que en sujetos consumidores de sustancias han sido consistentemente detectados diversos déficits cognitivos, apenas existen estudios relacionados con la anosognosia en estos pacientes. Shelton y Parsons (1987) mostraron que las auto-evaluaciones de deterioro cognitivo de alcohólicos recientemente abstinentes no estaban generalmente relacionadas con el rendimiento neuropsicológico objetivo, pero sí con medidas de estrés afectivo. Errico et al.(1990) mostraron que las estimaciones de alcohólicos respecto a sus propios déficits estaban más relacionadas con medidas de depresión y ansiedad que con las medidas neuropsicológicas objetivas. Horner, Harvey y Denier (1999) sugirieron también la existencia de ausencia de conciencia de los déficits en sujetos consumidores de drogas que iniciaban tratamiento.

Los problemas de control de impulsos han sido asimismo consistentemente registrados en sujetos consumidores de sustancias, tanto en paradigmas de descuento asociado al retraso (delay discounting), en los que el valor subjetivo de la recompensa decrece en función de la latencia del intervalo hasta su obtención (Green, Fry y Myerson, 1994; Petry y Casarella, 1999), como en tareas de toma de decisiones, como la Gambling Task, sensibles al daño en regiones prefrontales del córtex ( Bechara et al., 2001; London et al., 2001; Rogers et al., 1999), como en estimaciones conductuales tales como la alta co-ocurrencia de trastornos asociados al juego patológico (Steinberg, Costeen y Rounsaville, 1992; Feigelman et al., 1995). Algunos autores (Gerbing et al., 1987) han asociado estos déficits a la pre-existencia, o bien al desarrollo, de un rasgo general de impulsividad caracterizado por orientación hacia el presente, habilidad disminuida para retrasar la gratificación, desinhibición conductual, asunción de riesgos, búsqueda de sensaciones, propensión al aburrimiento, sensibilidad a la recompensa, hedonismo y pobre planificación, todas ellas manifestaciones características de disfunciones en el córtex prefrontal. Estas disfunciones parecen ser en numerosos casos responsables de la perseveración en el consumo y de la alta probabilidad de recaídas, por lo que deben ser convenientemente atajadas durante el proceso de rehabilitación.

\section{CONCLUSIONES Y LÍNEAS FUTURAS DE IN- VESTIGACIÓN.}

La investigación de los deterioros neuropsicológicos asociados al consumo crónico de drogas ha evolucionado en los últimos años desde el estudio de un amplio espectro de funciones que aparecían más o menos dañadas en función del tipo de sustancia consumida hasta el estudio de funciones específicas que se han mostrado consistentemente deterioradas como consecuencia del consumo de diversas sustancias y que presentan especial relevancia para el funcionamiento diario del sujeto consumidor, la práctica clínica, el éxito del tratamiento y la reincorporación del sujeto a su entorno bio-psico-social premórbido en unas condiciones óptimas de competencia y afrontamiento.

Entre los estudios clásicos, destacan los hallazgos neuropsicológicos que han contribuido a delimitar la presencia de un perfil de deterioro relativamente estable en consumidores de sustancias específicas. Se ha demostrado la existencia de déficits atencionales (atención selectiva y dividida) y memorísticos (memoria a corto plazo) en consumidores crónicos y severos de cannabis, de déficits en memoria a corto plazo, concentración, flexibilidad y control mental en consumidores crónicos de cocaína, de déficits en funciones memorísticas y ejecutivas y alteraciones de personalidad en consumidores recreativos de MDMA y de razonamiento abstracto y funciones ejecutivas en consumidores de opiáceos (heroína y morfina). Estas funciones se han mostrado nítidamente afectadas por el consumo crónico de drogas, y a pesar de que se produce cierto grado de recuperación con la abstinencia, algunos de estos deterioros pueden persistir muy a largo plazo. Desde una perspectiva clínica, la presencia de estos déficits puede contribuir a una deficiente asimilación de las normas, filosofía y contenidos de los programas de tratamiento.

Entre los estudios más recientes, destacan los avances producidos en la investigación de los deterioros de las funciones ejecutivas de los lóbulos prefrontales, que se han mostrado consistentemente afectadas por el consumo de diversas drogas: cannabis, cocaína, anfetaminas y heroína. Estas funciones son de crucial importancia, ya que constituyen el nivel superior de organización cerebral e implican mecanismos de control de impulsos y procesos de toma de decisiones que pueden condicionar el funcionamiento diario del sujeto, comprometer su capacidad de autocontrol y resistencia a posibles estresores relacionados o no con el consumo e incrementar el riesgo de recaídas. Asimismo, destaca la aplicación de nuevas herramientas de investigación (análisis de metabolitos, técnicas de neuroimagen funcional de última generación: PET, SPECT) al estudio de los mecanismos y regiones cerebrales implicadas en los deterioros neuropsicológicos asociados al consumo. Estos estudios han sugerido la existencia de diversas vías de acción (alteraciones estructurales, metabólicas y vasculares) a través de las cuales las drogas ejercerían sus efectos neurotóxicos sobre regiones corticales (córtex orbitofrontal) y subcorticales (núcleo accumbens, estriatum dorsal, núcleo mediodorsal del tálamo, giro cingulado, hipocampo) del cerebro, generando deterioros en un 
amplio espectro de funciones entre las que destacan la memoria y las funciones ejecutivas.

La convergencia entre los resultados de los estudios neuropsicológicos clásicos y los nuevos hallazgos en funciones ejecutivas derivados del desarrollo de nuevos modelos teóricos y tareas experimentales, y del uso de técnicas de neuroimagen y análisis metabólicos permiten sugerir que los deterioros en atención (selectiva y dividida), memoria y funciones ejecutivas (déficits de planificación, autorregulación, flexibilidad cognitiva, control de impulsos y toma de decisiones) son los más relevantes para la práctica clínica con drogodependientes, ya que pueden comprometer su capacidad de aprendizaje y asimilación de los contenidos y objetivos de los programas terapéuticos que actualmente se llevan a cabo en adicciones, su habilidad para manejar contingencias de reforzamiento y castigo, su capacidad para regular su propia conducta en función de metas deseables, la calidad y conveniencia de sus decisiones y, por tanto, el control de posibles recaídas.

Entre las consideraciones más importantes que se derivan del estudio de los correlatos neuropsicológicos del abuso de drogas para la práctica clínica con sujetos consumidores, dado que el estatus neuropsicológico parece tener un papel mediador sobre los resultados del tratamiento, destacan, en primer lugar, la potencial reversibilidad de los deterioros en función de la prolongación del periodo de abstinencia. Esta posibilidad de recuperación, que parece más evidente en consumidores más jóvenes y menos severos, pone de manifiesto, no obstante, la conveniencia de utilizar estrategias de rehabilitación neuropsicológica específicas que potencien tanto el número de funciones recuperadas como el grado de recuperación de las mismas, y alargar, en lo posible, la duración de los programas de tratamiento y rehabilitación, puesto que sólo así podremos aprovechar los evidentes beneficios de esta eventual recuperación de funciones.

Si bien no disponemos aún de protocolos específicos de rehabilitación neuropsicológica de las funciones más consistentemente deterioradas en sujetos drogodependientes, la existencia de un perfil de deterioro neuropsicológico distintivo y bien delimitado en esta población, la eficacia mostrada por estas técnicas neuropsicológicas en otras poblaciones clínicas, y las múltiples posibilidades de intervención apuntadas por la literatura disponible alientan el desarrollo y la incorporación de estos protocolos a la práctica clínica con consumidores de drogas. Estas estrategias de rehabilitación deben intervenir específicamente sobre aquellas funciones neuropsicológicas que se han mostrado más directamente asociadas al funcionamiento diario del drogodependiente, a su perseveración en el consumo y a la alta probabilidad de recaer, entre ellas la anosognosia o falta de conciencia acerca de su propio problema, la incapacidad para evocar respuestas emocionales ante potenciales situaciones de castigo, los déficits en los mecanismos de control de impulsos, y la deficiente calidad de sus procesos de toma de decisiones.

Estos estudios, han puesto asimismo de manifiesto, la necesidad de considerar la co-existencia de trastornos de personalidad (trastorno antisocial de la personalidad, trastorno obsesivo-compulsivo, esquizofrenia y síntomas psicóticos) y del estado de ánimo (depresión y ansiedad) que pueden potenciar la profusión y el grado de deterioro neuropsicológico y su influencia negativa sobre los resultados del tratamiento. Estos trastornos deben ser selectivamente abordados durante el tratamiento mediante estrategias de rehabilitación específicas que pueden beneficiarse, en algunos casos, del uso de tratamientos farmacológicos.

Aunque se ha avanzado considerablemente en la investigación neuropsicológica del abuso de sustancias, aún quedan por dilucidar muchas cuestiones en torno a los mecanismos a través de los cuales las drogas ejercen su influencia en diversas áreas del cerebro, tanto los datos de estudios neuropsicológicos y de neuroimagen como los procedentes de estudios y modelos animales podrían contribuir a completar el mapa de los mecanismos, regiones y funciones afectadas.

Por otra parte, la aparente reversibilidad de los déficits no está aún bien delimitada en el tiempo, no conocemos los efectos diferenciales de la rehabilitación neuropsicológica respecto a la recuperación espontánea y tampoco podemos precisar qué déficits recuperan con más rapidez, qué déficits tardan más tiempo en recuperar y cuáles carecen de posibilidades de recuperación.

Otro interesante campo de investigación es el de la comorbilidad y la dirección causa-efecto: si son alteraciones neurológicas leves durante el desarrollo las que están a la base de los rasgos de personalidad que predisponen al consumo o si el consumo de drogas puede inducir un deterioro neuropsicológico capaz de producir trastornos de la personalidad o del estado de ánimo son cuestiones aún sin dilucidar.

Por último, y de especial relevancia, será el estudio de la influencia del deterioro en funciones ejecutivas de planificación, secuenciación, control de impulsos y toma de decisiones sobre el funcionamiento diario del drogodependiente, su estilo de pensamiento, sus reacciones emocionales y sus posibilidades de reincorporación al entorno social de referencia en condiciones óptimas de funcionamiento y mínimas de riesgo.

\section{REFERENCIAS}

Aasly, J., Storsaeter, O., Nilsen, G., Smevik, O. y Rinck, P. (1993). Minor structural brain changes in young drug abusers. Acta Neurologica Scandinavica, 87, 210214. 
Adamse, M.A. (1987). Neuropsychological examination of a chronic cocaine abuse population. Dissertation Abstracts International, 48, 3143.

Allen, D.N. y Landis, R.K.B. (1998). Neuropsychological correlates of substance use disorders. En Clinical Neuropsychology (American Psychological Association: Washington D.C.), pp 591-612.

Allison, W.M. y Jerrom, D.W.A. (1984). Glue sniffing: A pilot study of three cognitive effects of long-term use. The International Journal of Addiction, 19 (4), 453-458.

Amass, L., Nardin, R., Mendelson, J.H., Teoh, S.K. y Woods, B.T. (1992). Quantitative magnetic resonance imaging in heroin- and cocaine-dependent men: a preliminary study. Psychiatry Research: Neuroimaging, 45, 15-23.

Ardila, A., Roselli, M. y Strumwasser, S. (1991). Neuropsychological effects of cocaine abuse. International Journal of Neurosciences, 57, 73-79.

Ardila, A. y Bateman, J.R. (1995). Psychoactive substance use: Some associated characteristics. Addictive Behaviors, 20, 249-254.

Arndt, S., Cohen, G., Alliger, R.J., Swayze II, V.W. y Andriasen, N.C. (1991). Problems with ratio and proportion measures of imaged cerebral structures. Psychiatry Research: Neuroimaging, 40, 79-89.

Azrin, R.L., Millsaps, C.L., Burton, D.B. y Mittenberg, W. (1992). Recovery of memory and intelligence following chronic cocaine abuse. Clinical Neuropsychology, 6 (3), 344.

Barkley, R.A., Fischer, M., Edelbrock, C.S. y Smallish, L. (1990). The adolescent outcome of hyperactive children diagnosed by research criteria, I: An 8-year prospective follow-up study. Journal of the American Academy of Child and Adolescent Psychiatry, 29, 546-557.

Barrat, E., Beaver, W., White, R., Blakeney, P. y Adams, P. (1972). The effects of of the chronic use of marijuana on sleep and perceptual motor performance in humans. En: Current Research in Marihuana (Lewis, M.F. ed.), pp. 163-193. Academic Press, New York.

Bartzokis, G., Beckson, M., Lu, P.H., Edwards, N., Rapoport, R., Wiseman, E. y Bridge, P. (2000). Age-related brain volume reductions in amphetamine and cocaine addicts and normal controls: implications for addiction research. Psychiatry Research: Neuroimaging, 98, 93-102.

Bauer, L.O., Hesselbrock, V.M., O'Connor, S. y Roberts, L. (1994a). P300 differences between non-alcoholic young men at average and above average risk for alcoholism: effects on distraction and task modality. Biological Psychiatry: Progress in Neuropsychofarmacology, 18, 263-277.

Bauer, L.O., O'Connor, S. y Hesselbrock, V.M. (1994b). Frontal P300 decrements in Antisocial Personality Disorder. Alcohol: Clinical and Experimental Research, 6, 1300-1305.

Bauer, L.O. (1997a). Frontal P300 decrements, childhood conduct disorder, family history, and the prediction of relapse among abstinent cocaine users. Drug and Alcohol Dependence, 44, 1-10.
Bauer, L.O. (1997b). Smooth pursuit eye movement dysfunction in abstinent cocaine abusers: Effects of a family history of alcoholism. Alcohol: Clinical and Experimental Research, 21, 910-915.

Bauer, L.O. y Hesselbrock, V.M. (1999). P300 decrements in teenagers with conduct problems: Implications for substance abuse risk and brain development. Biological Psychiatry, 46, 263-272.

Bauer, L. (2001). Antisocial personality disorder and cocaine dependence: their effects in behavioural and electroencephalographic measures of time estimation. Drug and Alcohol Dependence, 63, 87-95.

Baxter, L.R.Jr., Schwartz, J.M., Phelps, M.E., Mazziota, J.C., Barrio, J., Rawson, R.A., Engel, J., Guze, B.H., Selin, C. y Sumida, R. (1988). Localization of neurochemical effects of cocaine and other stimulants in human brain. Journal of Clinical Psychiatry, 49, 23-26.

Beatty, W.W., Katzun, V.M., Moreland, V.J. y Nixon, S.J. (1995). Neuropsychological performance of recently abstinent alcoholics and cocaine abusers. Drug and Alcohol Dependence, 37, 247-253.

Beatty, W.W. y Borrell, G.K. (2000). Forms of knowledge, cognitive impairment and drug abuse: a demonstration. Neuropsychopharmacology and Biological Psychiatry, $24,17-22$

Bechara, A., Damasio, H. y Damasio, A.R. (2000). Emotion, decision making and the orbitofrontal cortex. Cerebral Cortex, 10, 295-307.

Bechara, A., Dolan, S., Denburg, N., Hindes, A., Anderson, S.W. y Nathan, P.E. (2001). Decision-making deficits, linked to a dysfunctional ventromedial prefrontal cortex, revealed in alcohol and stimulant abusers. Neuropsychologia, 39, 376-389.

Bennet, T.L. (1987). Individual psychotherapy and minor head injury. Cognitive Rehabilitation, 3, 20-24.

Berry, G.J., Heaton, R. y Kirby, M. (1978). Neuropsychological assessment of chronic inhalant abusers: A preliminary report. En: C.W. Sharp y L.T. Carroll (Eds.). Voluntary inhalation of industrial solvents (pp 111-136). Rockville, MD: National Institute of Drug Abuse.

Berry, J., Van Gorp, W.G., Herzberg, D.S. y Hinkin, C. (1993) Neuropsychological deficits in abstinent cocaine abusers: Preliminary findings after two weeks of abstinence. Drug and Alcohol Dependence, 32, 231237.

Biederman, J., Farone, S., Milberger, S., Guite, J., Mick, E., Chen, L., Mennin, D., Marrs, A., Oullette, C., Moore, P., Spencer, T., Norman, D., Wilens, T., Kraus, I. Y Perrin, J. (1996). A prospective 4-years follow-up study of attention-deficit hyperactivity and related disorders. Archives of General Psychiatry, 53, 437-446.

Bigler, E.D. (1979). Neuropsychological evaluation of adolescent patients hospitalised with chronic inhalant abuse. Clinical Neuropsychology, 1, 8-12.

Block, R.I. y Ghoneim, M.M. (1993) Effects of Chronic marijuana use on human cognition. Psychopharmacology, $110,219-228$. 
Bolla, K., McCann, U. y Ricaurte, G. (1998). Impaired memory function in abstinent 3,4-methylenedioxymetanphetamine (MDMA, "ecstasy") users. Neurology, 51, 1532-1537.

Bremner, J.D., Innis, R.B., Salomon, R.M., Staib, L.H., Ng, C.K., Miller, H.L., Bronen, R.A., Krystal, J.H., Duncan, J., Rich, D., Price, L.H., Malison, R., Dey, H., Soufer, R. y Charney, D.S. (1997). Positron emission tomography measurement of cerebral metabolic correlates of tryptophan depletion induced depressive relapse. Archives of General Psychiatry, 54, 364-374.

Brook, J.S., Whiteman, M., Finch, S. y Cohen, P. (1995b). Aggression, intrapsychic distress, and drug use: Antecedents and intervening processes. Journal of the American Academy of Child and Adolescent Psychiatry 34, 1076-1084

Bruhn, P. y Maage, N. (1975). Intellectual and neuropsychological functions in young men with heavy and long-term patterns of drug abuse. American Journal of Psychiatry, 132, 397-401.

Cacciola, J.S., Alterman, A.I., Rutherford, M.J., McKay y Snider, E.C. (1996). Personality disorders and treatment outcome in methadone maintenance patients. Journal of Nervous Mental Disease, 184, 234-239.

Campodonico, J.R. y McGlynn, S.M. (1995). Assessing awareness of deficits: recent research and applications. En: Cushman, L.A., Scherer, M.J. (Eds.), Measurement and Instrumentation in Psychology. Psychological Assessment in Medical Rehabilitation. American Psychological Association Books, Washington D.C., pp: 393-418.

Cassidy, G. y Ballard, C. (1994). Psychiatric sequelae of MDMA (ecstasy) and related drugs. Irish Journal of Psychological Medicine, 11, 132-133.

Chait, L.D. y Pierri, J. (1992). Effects of smoked marijuana on human performance: A critical review. En: Murphy, L., Bartke,A.;eds.Marihuana/cannabinoids: Neurobiology and Neurophysiology. Boca Ratón, FL: CRC Press; 1992: 387-423.

Cloninger, C.R. (1997). Neurogenetic adaptive mechanisms in alcoholism. Science, 236, 410-436.

Cohen, S., Lessin, P.J., Hahn, P.M. y Tyrrell, E.D. (1976b). A 94-day cannabis study. En: The Pharmacology of Marijuana (Braude, M.C. y Szara, S., eds), pp. 621-626. Raven Press, New York.

Creighton, F., Black, D. y Hyde, C. (1991). "Ecstasy" psychosis and flashbacks. British Journal of Psychiatry, 159, 713-715.

Crits-Cristoph, P., Siqueland, L., Blaine, J, Frank, A., Luborsky, L., Onken, L.S. et al. (1999). Psychosocial treatments for cocaine dependence: NIDA collaborative cocaine treatment study. Archives of General Psychiatry, 56, 493-502.

Culver C.M. y King, F.W. (1974). Neuropsychological assessment of undergraduate marijuana and LSD users. Archives of General Psychiatry, 31, 707-711

Dawes, M.A., Tarter, R.E. y Kirisci, L. (1997). Behavioral self regulation: Correlates and 2 year follows-ups for boys at risk for substance abuse. Drug and Alcohol Dependence, 45, 165-176.
Di Chiara, G. (1999). Drug addiction as dopamine dependent associative learning disorder. European Journal of Pharmacology, 375, 13-30.

Dornbush, R.L., Clare, G., Zaks, A., Crown, P., Volavska, J. y Fink, M. (1972). 21-day administration of marijuana in male volunteers. En : Current Research in Marijuana (Lewis, M.F., ed.), pp. 115-128. Academic Press, New York.

Easton, C. y Bauer, L.O. (1996). Neuropsychological correlates of urine toxicology results. Neuropsychopharmachology and Biological Psychiatry, 20, 969982.

Ebmeier, K.P., Cavanagh, J.T., Moffoot, A.P., Glabus, M.F., O'Carroll, M.E. y Goodwin, G.M. (1997). Cerebral perfusion correlates of depressed mood. British Journal of Psychiatry, 170, 77-81.

Ehrenreich, H., Rinn, T., Kunert, H.J., Moeller, M.R., Poser, W., Schilling, L., Gigerenzer, G., Hoehe, M.R. (1999). Specific attentional dysfunction in adults following early start of cannabis use. Psychopharmachology, 142, 295-301.

Ellenberg, L., Roosenbaum, G., Goldman, M., y Whitman, R.D. (1980). Recoverability of psychological functioning following alcohol abuse: Lateralization effects. Journal of Consulting and Clinical Psychology, 48, 503-510.

Errico, A.L., Nixon, S.J., Parsons, O.A. y Tassey, J. (1990). Screening for neuropsychological impairment in alcoholics. Psychological Assessment, 2, 45-50.

Fals-Stewart, W. (1992). Personality characteristics of substance abusers: An MCMI cluster typology of recreational drug users treated in a therapeutic community and its relationship to length of stay and outcome. Journal of Personality Assessment, 59, 515-527.

Fals-Stewart, W. (1993). Neurocognitive defects and their impact on substance abuse treatment. Journal of Addictions and Offender Counselling, 13, 46-57.

Fals-Stewart, W. y Lucente, S. (1994a). The effect of neurocognitive status and personality funtioning on length of stay in residential substance abuse treatment: An integrative study. Psychology of Addictive Behaviors, 8, 1-12.

Fals-Stewart, W. y Lucente, S. (1994b). The effect of cognitive rehabilitation on the neuropsychological status of patients in drug abuse treatment who display neurocognitive impairment. Rehabilitation Psychology, 39, 79-94.

Feigelman, W., Kleinman, P.H., Lesieur, H.R., Millman, R. y Lesser, M. (1995). Pathological gambling among methadone patients. Drug and Alcohol Dependence, 39, 75-81.

Fields, S. y Fullerton, J. (1975). Influence of heroin addiction in neuropsychological functioning. Journal of Clinical and Consulting Psychology, 43, 114.

Fletcher, J.M., Page, B, Francis, D.J., Copeland, K., Naus, M.J., Davis, C.M., Morris, R., Krauskopf, D. Satz, P. (1996). Cognitive correlates of long-term cannabis use in Costa Rica men. Archives of General Psychiatry, $53,1051-1057$. 
Frank, I.M., Lessin, P.J., Tyrrell, E.D., Hahn, P.M. y Szara, S. (1976). Acute and cumulative effects of marijuana smoking in hospitalized subjects: a 36-day study. En : The Pharmacology of Marijuana (Braude, M.C. y Szara, S., eds), pp. 673-679. Raven Press, New York.

Galloway, M.P. (1988). Neurochemical interactions of cocaine with dopaminergic systems. Trends in Pharmacological Science, 9, 452-454.

Gawin, F.H y Kleber, H.D. (1986). Abstinence symptomatology and psychiatric diagnosis in cocaine abusers. Archives of Clinical Psychiatry, 43, 107-113.

Gerbing, D.W., Ahadi, S.A. y Patton, J.H. (1987). Toward a conceptualization of impulsivity: Components across the behavioural and self-reports domains. Multivariate Behavioral Research, 22, 357-379.

Gerra, G., Calbiani, B., Zaimovic, A., Sartori, R., Ugolotti, G., Ippolito, L., Delsignore, R., Rustichelli, P. y Fontanesi, B. (2000). Regional cerebral blood flow and comorbid diagnosis in abstinent opioides addicts. Psychiatry Research: Neuroimaging, 83, 117-126.

Giancola, P.R., Martín, C.S., Tarter, R.E., Moss, H.B. y Pelham, W.E. (1996). Executive cognitive function and aggressive behavior in preadolescent boys at high risk for substance abuse. Journal of Studies on Alcohol, 57, 352-359.

Gillen, R.W., Kranzler, H.R., Bauer, L.B., Burleson, J.A., Samarel, D. y Morrison, D.J. (1998). Neuropsychological findings in cocaine-dependent outpatients. Progress in Neuropsychopharmacology and Biological Psychiatry, 22, 1061-1076.

Glenn, S.W., Parsons, O.A., Sinha, R., y Stevens, L. (1988). The effects of repeated withdrawals from alcohol on the memory of male and female alcoholics. Alcohol and Alcoholism, 23, 337-342.

Goldberg, T.E., Bigelow, L.B., Weinberger, D.R., Daniel, D.G. y Kleinman, J.E. (1991). Cognitive and behavioural effects on coadministration of dextroanphetamine and haloperidol in schizophrenia. American Journal of Psychiatry, 148, 78-84.

Goldman, M. (1983). Cognitive impairment in chronic alcoholics: Some cause for optimism. American Psychologist, 38, 1045-1054.

Goldman, M., Williams, D. y Klisz, D. (1983). Recoverability of motor and sensory function following chronic alcohol abuse: Prolonged visual-spatial dysfunction in older alcoholics. Journal of Consulting and Clinical Psychology

Grant, I., Rochford, J., Fleming, T. y Stunkard, A. (1973). A neuropsychological assessment of the effects of moderate marijuana use. Journal of Nervous Mental Diseases, 156 (4), 278-280.

Grant, I. y Judd, L.L. (1976). Neuropsychological and EEG disturbances in polydrug users. American Journal of Psychiatry, 133, 1039-1042.

Grant, I., Adams, K.M., Carlin, A.S., Rennick, P.M., Judd, L.L.y Schoof, K. (1978). The collaborative neuropsychological study of polydrug users. Archives of General Psychiatry, 35, 1063-1075.
Grant, S., Conttoreggi, C. y London, E.D. (2000). Drug abusers show impaired performance in a laboratory test of decision-making. Neuropsychologia, 38, 11801187.

Green, L., Fry, A.F. y Myerson, J. (1994). Discounting of delayed rewards: a life span comparison. Psychological Science, 5, 33-36.

Grewal, R. y Miller, B.L. (1991). Cocaine induced hypertensive encephalopathy. Acta Neurologica, 13, 279-281.

Gunnarsdottir, E.D., Pingitore, R.A., Spring, B.J., Konopka, B.N., Crayton, J.W., Milo, T. y Shirazi, P. (2000). Individual differences among cocaine users. Addictive Behaviors, 25 (5), 641-652.

Gyulai, L., Alavi, A., Broich, K., Reilley, J., Ball, W.B. y Whylbrow, P.C. (1997). I-123 iofetamine single-photon emission tomography in rapid cycling bipolar disorder: a clinical study. Biological Psychiatry, 41, 152-161.

Hanks, G. W., O’Neill, W.M., Simpson, P. y Wesnes, K. (1995). The cognitive and psychomotor effects of opioids analgesics. II. A randomized controlled trial of single doses of morphine, lorazepam and placebo in healthy adults. European Journal of Clinical Pharmacology, 48, 455-460.

Heishman, S.J., Arasteh, K. y Stitzer, M.L. (1996). Comparative effects of alcohol and marijuana on mood, memory and performance. Pharmacology, Biochemistry and Behavior, 58 (1), 93-101.

Henry, B., Caspy, A., Moffitt, T.E. y Silva, P.A. (1996). Temperamental and familial predictors of violent and non-violent criminal convictions: Age 3 to age 18 . Developmental Psychology, 32, 614-623.

Hill, S.Y. y Mikhael, M.A. (1979). Computerised transaxial tomographic and neuropsychological evaluations in chronic alcohol and heroin abusers. American Journal of Psychiatry, 136, 598-602

Hill, S.Y., Reyes, R.B., Mikhael, M. y Ayre, F. (1979). A comparison of alcoholics and heroin users: Computerised transaxial tomography and neuropsychological functioning. Currents in Alcoholism, 5, 187-205

Hoff, A.L., Riordan, H., Alpert, R. Y Volkow, N. (1991). Cognitive function in chronic cocaine abusers. Journal of Clinical and Experimental Psychology, 13, 60

Hoover, A. (1991). Problems of alcohol and another drug use and abuse in adolescents. Journal of Adolescent Health, 12, 606-613.

Horner, M.D. (1997). Cognitive functioning in alcoholic patients with and without cocaine dependence. Archives of Clinical Neuropsychology, 12 (7), 667676

Horner, M.D., Harvey, R.T. y Denier, C.A. (1999). Self-report and objective measures of cognitive deficit in patients entering substance abuse treatment. Psychiatry Research, 86 (2), 155-161.

Huttenlocher, P.R. (1990). Morphometric study of human cerebral cortex development. Neuropsychologia, 28, 517-527. 
Hyman, S.E. y Malenka, R.C. (2001). Addiction and the brain: the neurobiology of compulsion and its persistence. Nature Reviews, 2, 695-703.

Jasiukaitis, P. y Fein, G. (1999). Intact visual word priming in cocaine dependent subjects with and without cognitive deficit. Neuropsychopharmachology and Biological Psychiatry, 23, 1019-1036.

Jones, R.T. y Benowitz, N. (1976). The 30-day trip -clinical studies of cannabis tolerance and dependence. En: The Pharmacology of Marijuana (Braude, M.C. y Szara, S., eds), pp. 627-642. Raven Press, New York.

Klisz, D.K. y Parsons, O.A. (1977). Hypothesis testing in younger and older alcoholics. Journal of Studies on Alcohol, 46, 116-121.

Kolb, B. y Fantie, B. (1989). Development of the child's brain and behavior. En: C.R. Reynolds y E. Fletcher-Janzen (Eds.), Handbook of Clinical Child Neuropsychology (pp. 17-39). New York: Plenium.

Kosten, T.R. (1998). Pharmacotherapy of cerebral ischemia in cocaine dependence. Drug and Alcohol Dependence, 49, 133-144.

Kouri, E.M., Lukas, S.E. y Mendelson, J.H. (1996). P300 assessment of opiate and cocaine users: effects of detoxification and buprenorphine treatment. Biological Psychiatry, 40, 617-628.

Kristal, J. y Price, L. (1992). Chronic 3,4-methylenedioxymetanphetamine ("ecstasy") in recreational users. Journal of Clinical Psychopharmacology, 11, 302305.

Krueger, R.F., Caspi, A., Moffit, T.E., Silva, P.A. y McGee, R. (1996). Personality traits are differentially linked to mental disorders: a multitrait-multidiagnosis study of adolescent birth cohort. Journal of Consulting and Clinical Psychology, 57, 698-704.

Kuruoglu, A.C., Arikan, Z., Vural, G., Karatas, M., Arac, M. y Isik, E. (1996). Single photon emission computerised tomography in chronic alcoholism. Antisocial personality disorder may be associated with decreased frontal perfusion. British Journal of Psychiatry, 169, 348-354.

Lawrence, A.D., Sahakian, B.J., Hodges, J.R., Rosser, A.E. y Robbins, T.W. (1996). Executive and mnemonic function in early Huntington's disease. Brain, 119, 1597-1613.

Leal, J., Ziedonis, D. y Kosten, T. (1994). Antisocial personality disorder as a prognostic factor for pharmacotherapy of cocaine dependence. Drug and Alcohol Dependence, 35, 31-35.

Leeds, N.E., Malhotra, V. y Zimmerman, R.D. (1983). The radiology of drug addiction affecting the brain. Seminars of Roentgenology XVIII, 227-233.

Levin, J.M., Holman, B.L., Mendelson, J.H., Teoh, S.K., Garada, B., Johnson, K.A. y Springer, S. (1994). Gender differences in cerebral perfusion in cocaine abuse: Technetium-99m-HMPAO SPECT study of drug abusing women. The Journal of Nuclear Medicine, 35, 19021909.

Littrell, K.H., Hilligoss, N.M., Peabody, C.D. y Johnson, C.G. (2001). Olanzapine treatment for patients with schizophrenia and substance abuse. Journal of Substance Abuse Treatment, 21, 217-221.

Liu, X., Matochick, J.A., Cadet, J. y London E.D. (1998). Smaller volume of prefrontal lobe in polysubstance abusers: a magnetic resonance imaging study. Neuropsychopharmacology, 18, 243-252.

London, E.D., Cascella, N.G., Wong, D.F., Phillips, R.L., Dannals, R.F., Links, J.M., Herning, R., Grayson, R., Jaffe, J.H. y Wagner, H.N. Jr. (1990). Cocaine induced reduction of glucose utilization in human brain. A study using positron emission tomography and (fluorine 18)fluorodexyglucose. Archives of General Psychiatry, 47, 567-574.

London, E.D., Ernst, M., Grant, S., Bonson, K. y Weinstein, A. (2000). Orbitofrontal cortex and human drug abuse: functional imaging. Cerebral Cortex, 10, 334-342.

Lundqvist, T. (1995). Specific thought patterns in chronic cannabis smokers observed during treatment. Life Sciences, 56 (23), 2141-2144.

Madden, G.J., Petry, N.M., Badger, G.J. y Bickel, W.K. (1997). Impulsive and self-control choices in opioid-dependet patients and non-drug-using control participants: drug and monetary rewards. Experimental Clinical Psychopharmacology, 5, 256-262.

Manschreck, T.C., Laughery, J.A., Weinstein, C.C., Humblestone, B., Neville, M., Podlewsky, H. y Mitra, N.(1988). Characteristics of freebase cocaine psychosis. Yale Journal of Biological Medicine, 61, 115-122.

Manschreck, T.C., Schneyer, M.L., Weinstein, C.C., Laughery, J., Rosenthal, J, Celada, T. y Berner, J. (1990). Freebase cocaine and memory. Comprehensive Psychiatry, 31, 369-375.

Marlowe, D.B., Kirby, K.C., Festinger, D.S., Husband, S.D. y Platt, J.J. (1997). Impact of comorbid personality disorders and personality disorders symptoms on outcomes of behavioural treatment for cocaine dependence. Journal of Nervous Mental Disease, 185, 483-490.

McCaffrey, R.J. (1989). Neuropsychological sequelae of polysubstance abuse in a sample of inpatient adolescent. Unpublished raw data.

McCaffrey, R.J. y Forneris, C.A. (1997). Adolescent substance abuse: A Biopsychosocial Perspective. En: Biological and Neuropsychological Mechanismn: Life-Span Developmental Psychology; Reese y Franzen (Eds.). Lawrence Erlbaum Associates, Inc. Mahwah: New Jersey.

McCann, U. y Ricaurte, G. (1991). Lasting neuropsychiatric sequelae of methylenedioxymetanphetamine ("ecstasy") in recreational users. Journal of Clinical Psychopharmacology, 11, 302-305.

McGlynn, S.M. y Schacter, D.L. (1989). Unawareness of deficits in neuropsychological syndromes. Journal of Clinical and Experimental Neuropsychology, 11, 143-205.

McGuire, P.K. y Fahy, T. (1991). Flashbacks following MDMA. British Journal of Psychiatry 160, 275-276. 
McGuire, P.K., Cope, H.M. y Fahy, T. (1994a). Diverse psychiatric morbidity associated with use of 3-4methylenedioxymethamphetamine ("ecstasy"). British Journal of Psychiatry, 165, 391-394.

McGuire, P.K., Jones, P., Bebbington, P., Toone, B.K., Lewis, S.W. y Murray, R.M. (1994b). Cannabis and acute psychosis. Schizophrenia Research, 13, 161-168.

McGuire, P. (2000). Long-term psychiatric and cognitive effects of MDMA use. Toxicology Letters, 112-113, 153-156.

McKetin, R. y Mattick, R.P. (1998). Attention and memory in illicit amphetamine users: comparison with non-drugusing-controls. Drug and Alcohol Dependence, 50, 181-184.

Mendelson, J.H., Babor, T.F., Kuehnle, J.C., Rossi, A.M., Bernstein, J.G., Mello, N.K. y Greenberg, I. (1976). Behavioral and biological aspects of marihuana use. Annual New York Academic Sciences, 282, 186-210

Mendhiratta, S.S., Wig, N.N. y Varma, V.K. (1978). Some psychological correlates of long-term heavy cannabis users. British Journal of Psychiatry, 132, 482-486.

Mendhiratta, S.S., Varma, V.K., Dang, R., Malhotra, A.K., Das, K. Y Nehra, R. (1988) Cannabis and cognitive functions: a re-evaluation study. British Journal of Addiction, 83, 749-753.

Miller, L. (1990). Neuropsychodynamics of alcoholism and addiction: Personality, psychopathology, and cognitive style. Journal of Substance Abuse Treatment, 7, 3149.

Mittenberg, W. y Motta, S. (1993). Effects of chronic cocaine abuse in memory and learning. Archives of Clinical Neuropsychology, 8, 477-483.

Mody, C.K., Miller, B.L., Mclntyre, H.B. et al. (1998). Neurological complications of cocaine abuse. Neurology, 38, 1189-1193.

Morgan, M.J., Cascella, N.G., Stapleton, J.M., Phillips, R.L., Yung B.C., Wong, D.F. et al.(1993). Sensitivity to subjective effects of cocaine in drug abusers: Relationship to cerebral ventricle size. American Journal of Psychiatry, 150, 1712-1717.

Morgan, M. (1998). Recreational use of MDMA ("ecstasy") is associated with elevated impulsivity. Neuropsychopharmacology, 19, 252-264.

Morgan, M. (1999). Memory deficits associated with recreational use of "ecstasy" (MDMA). Psychopharmacology, 141, 30-36.

Moss, H., Blackson, T., Martin, C. y Tarter, R. (1992). Heightened motor activity level in male offspring of substance abusing fathers. Biological Psychiatry, 32, 1135-1147.

Mozley, P.D., Hornig Rohan, M., Woda, A.M., Kim, H.J., Alavi, A., Payer, F. y Amsterdam, J.D. (1996). Cerebral HMPAO SPECT in patients with major depression and healthy volunteers. Progress in Neuropsychofarmacology and Biological Psychiatry, 20, 443-458.

Newcomb, M.D. y Bentler, P.M. (1988). Consequence of adolescent drug use: Impact on the lives of young adults. Newbury Park, CA: Sage.
O'Carroll, R.E. y Goodwin, G.M. (1997). Cerebral perfusion correlates of depressed mood. British Journal of Psychiatry, 170, 77-81.

O'Connor, S., Bauer, L.O., Tasman, A. y Hesselbrock, V.M. (1994). Reduced P3 amplitudes of ERPs are associated with both a family history of alcoholism and antisocial personality disorder. Biological Psychiatry: Progress in Neuropsychofarmacology, 18, 1307-1321.

O’Malley, S., Adamse, M., Heaton, R.K. y Gawin, F.H. (1990). Neuropsychological impairment in chronic cocaine abusers. American Journal of Drug and Alcohol Abuse, 18, 131-144.

O'Malley, S.S. y Gawin, F.H. (1990). Abstinence symptomatology and neuropsychological impairment in chronic cocaine abusers. En: Residual Effects of Abused Drugs in Behavior, NIDA Research Monograph, 101 (Spencer, J.W. y Boren, J.J., eds.), pp. 179-190. US Department of Health and Human Services, Rockville, MD.

Ornstein, T.J., Iddon, J.L., Baldacchino, A.M., Sahakian, B.J., London, M., Everitt, B.J. y Robbins, T.W. (2000). Profiles of cognitive dysfunction in chronic amphetamine and heroin abusers. Neuropsychopharmacology, 23(2), 113-126.

Owen, A.M., Roberts, A.C., Polkey, C.E., Sahakian, B.J. y Robbins, T.W. (1991). Extra-dimensional versus intradimensional set-shifting performance following frontal lobe excisions, temporal lobe excisions or amygdalahippocampectomy in man. Neuropsychologia, 29 (10), 993-1006.

Page, J.B., Fletcher, J. y True, W.R. (1988). Psychosociocultural perspectives on chronic cannabis use: The Costa Rican follow up. Journal of Psychoactive Drugs, 20 (1), 5765.

Pallanti, S. y Mazzi, D. (1992). MDMA ("ecstasy") precipitation of panic disorder. Biological Psychiatry, 32, 91-95.

Parrot, A., Lees, A., Garnham, N., Jones, M. Y Wesnes, K. (1998). Cognitive performance in recreational users of MDMA or "ecstasy": evidence for memory deficits. Journal of Psychopharmacology, 12, 79-83.

Parsons, O.A. y Leber, W.R. (1982). Alcohol, cognitive dysfunction and brain damage. En: National Institute on Alcohol Abuse and Alcoholism (Ed.) Biomedical processes and consequences of alcohol use (Alcohol and Health Monograph 2, DHHS Publication No ADM 82-1191, pp 213-253). Washington D.C.: US Government Printing Office.

Pascual-Leone, A., Dhuna, A. y Anderson, D.C. (1991). Cerebral atrophy in habitual cocaine abusers: a plannimetric CT study. Neurology, 4, 34-38.

Pearlson, G.D., Jeffery, P.J., Harris, G.J., Ross, C.A., Fishman, M.W. y Camargo, E.E. (1993). Correlation of acute cocaine-induced changes in local cerebral blood flow with subjective effects. American Journal of Psychiatry, 150, 495-497.

Petry, N.M, Bickel, W.K. y Arnett, M. (1998). Shortened time horizons and insensivity to future consequences in heroin addicts. Addiction, 93, 729-738. 
Petry, N.M. y Casarella, T. (1999). Excessive discounting of delayed rewards in substance abusers with gambling problems. Drug and Alcohol Dependence, 56, 25-32.

Pezawas, L.M., Fischer, G., Diamant, K., Schneider, C., Schindler, S.D., Thurnher, M, Ploechl, W., Eder, H. y Kasper, S. (1998). Cerebral CT findings in male opioiddependent patients: Stereological, planimetric and linear measurements. Psychiatry Research: Neuroimaging, 83, 139-147.

Pogge, D.L., Horan, W.P., Borgaro, S.R., Stokes, J.M., Lord, J.J. y Harvey, P.D. (1996). Cognitive impulsivity in adolescent conduct disorder and depression. Biological Psychiatry, 39, 536.

Pogge, D.L., Horan, W.P., Borgaro, S.R., Stokes, J.M., Lord, J.J. y Harvey, P.D. (1996). Conduct disorder, impulsivity and substance abuse in adolescents. Biological Psychiatry, 39, 536-537.

Pontius, A.A. y Ruttiger, K.F. (1976). Frontal lobe system maturational lag in juvenile delinquents shown in narrative test. Adolescence, 11, 509-518.

Pope Jr., H.G., Gruber, A.J., Yurgelun-Todd, D. (1995). The residual neuropsychological effects of cannabis: the current status of research. Drug and Alcohol Dependence, 38, 25-34.

Post, R.M. (1975). Cocaine psychoses: A continuum model. American Journal of Psychiatry, 132, 225-231.

Press, R.J. (1983). The neuropsychological effects of cocaine and opiate use. Ann Arbor, MI: University Microfilms International.

Prigatano, G.P. (2001). Rehabilitation of higher cerebral functions and the patient's personality. BNI Quarterly (in press).

Rawson, R.A., Engel, J., Guze, B., Selin, C. y Sumida, R. (1988). Localization of neurochemical effects of cocaine and other stimulant in the human brain. Journal of Clinical Psychiatry, 49, 23-26.

Reich, J.H. y Vasile, R.G. (1993). Effect of personality disorders on the treatment outcome of Axis I conditions: an update. Journal of Nervous Mental Disease, 181, 247-291

Riggs, D., Langenbucher, J., Goldman, M. y Brown, S. (2000). Adolescents are not adults: developmental considerations in alcohol users. Alcoholism, Clinical and Experimental Research, 24 (2), 232-237.

Robinson, T.E. y Kolb, B. (1999). Alterations in the morphology of dentrites and dentritic spines in the nucleus accumbens and prefrontal cortex following repeated treatment with amphetamine and cocaine. European Journal of Neurosciences, 11, 1598-1604.

Rockstroh, B., Elbert, T., Birbaumer, M. y Lutzenbereger, W. (1982). Slow brain potentials and behaviors. Urban y Schwarzenberg: Baltimore.

Rodríguez-Álvarez, M. (1981). Evaluación neuropsicológica de la memoria viso-constructiva inmediata en heroinómanos. Psiquis, 12, 55-62.

Rogers, R.D., Everitt, B.J., Baldacchino, A., Blackshaw, A.J., Swainson, R., Wynne, K., Baker, N.B., Hunter, J., Carthy, T., Booker, E., London, M., Deakin, J.F.W, Sahakian, B.J. y Robbins, T.W. (1999). Dissociable deficits in the decision-making cognition of chronic anphetamine abusers, opiate abusers, patients with focal damage to prefrontal cortex, and tryptophan-depleted normal volunteers: evidence for monoaminergic mechanisms. Neuropsychopharmacology, 20 (4), 322-339.

Rogers, R.D. y Robbins, T.W. (2001). Investigating the neurocognitive deficits associated with chronic drug misuse. Current Opinion in Neurobiology, 11, 250257.

Rose, J.S., Branchey, M., Buydens-Branchey, Stapleton, J.M., Chasten, K., Werrell, A. y Maayan, M.L. (1996). Cerebral perfusion in early and late opiate withdrawal: a technetium-99-m-HMPAO SPECT study. Psychiatry Research: Neuroimaging, 67, 39-47.

Roselli, M. y Ardila, A. (1996). Cognitive effects of cocaine and polydrug abuse. Journal of Clinical and Experimental Neuropsychology, 18 (1), 122-135.

Rounsaville, B., Novelly, R., Kleber, H. y Jones, C. (1981). Neuropsychological impairment in opiate addicts: Risk Factors. En: R.B. Millman, P. Cushman y J.H. Lowison (Eds.). Research developmentes in drug and alcohol abuse (pp 79-90). New York: New York Academy of Sciences.

Rounsaville, B.J., Foley, S., Carroll, K., Budde, D., Prusoff, B.A. y Gawin, F. (1991). Psychiatric diagnoses of treatment seeking cocaine abusers. Archives of Clinical Psychiatry, 48, 43-51.

Rourke, B.P., Bakker, D.J., Fisk, J.L., y Strang, J.D. (1983). Child neuropsychology: An introduction to theory, research, and clinical practice. New York: Guilford.

Schaefer, J., Andrysiak, T. y Ungerleider, J.T. (1981). Cognitive and long-term use of ganja (cannabis). Science, 213, 465-466.

Schau, E.J., O'Leary, M.R. y Chaney, E.F. (1980). Reversibility of cognitive deficits in alcoholics. Journal of Studies on Alcohol, 41, 733-740.

Schifano, F. (1991). Chronic atypical psychosis associated with MDMA ("ecstasy") abuse. Lancet, 338, 1335.

Schwartz, R.H., Gruenewald, P.J., Klitzner, M. y Fedio, P. (1989). Short-term memory impairment in cannabisdependent adolescents. American Journal of Diseases in Childhood, 143, 1214-1219.

Scott, J., Gilvarry, E. y Farrell, M. (1998). Managing anxiety and depression in alcohol and drug dependence. Addictive Behaviors, 23 (6), 919-931.

Selby, M.J. y Azrin, R.L. (1998). Neuropsychological functioning in drug abusers. Drug and Alcohol Dependence, 50 (1), 39-45.

Serper, M.R., Copersino, M.L., Richarme, D., Vadhan, N. y Cancro, R. (2000). Neurocognitive functioning in recently abstinent cocaine-abusing schizophrenic patients. Journal of Substance Abuse, 11 (2), 205-213.

Shelton, M.D. y Parsons, O.A. (1987). Alcoholics selfassessment of their neuropsychological functioning in every day life. Journal of Clinical Psychology, 43, 395-403.

Sklair-Tavron, L., Shi, W., Lane, S.B., Harris, H.W., Bunney, B.S. y Nestler, E.J. (1996). Chronic morphine induces visible changes in the morphology of mesolimbic 
dopamine neurons. Proceedings of the National Academy of Sciences, USA, 93, 11202-11207.

Skodol, A.E., Oldham, J.M. y Gallaher, P.E. (1999). Axis II comorbidity of substance use disorders among patients refered for treatment of personality disorders. American Journal of Psychiatry, 156, 733-738.

Sohlberg, M.M. y Mateer, C.A. (1989). Introduction to cognitive rehabilitation. New York: Guilford.

Solowij, N., Hall, W. Y Lee, N. (1992). Recreational MDMA use in Sydney: a profile of "ecstasy" users and their experiences with the drug. British Journal of Addiction, 87, 1161-1172.

Solowij, N. (1995). Do cognitive impairments recover following cessation of cannabis use. Life Sciences, 56, 2119-2126.

Solowij, N., Mitchie, P.T. y Fox, A.M. (1995). Differential impairments of selective attention due to frequency and duration of cannabis use. Biological Psychiatry, 37, 731-739.

Souief, M.I. (1976b). Differential associations between chronic cannabis use and brain function deficits. Annual New York Academic Sciences, 282, 323-343.

Spencer, J. (ed.) (1991). The residual effects of abused drugs on performance. US Superintendent of Documents, Washington D.C.

Stapleton, J.M., Morgan, M.J., Phillips, R.L., Wong, D.F., Yung, B.C.K., Shaya, E.K., Dannals, R.F., Liu, X., Grayson, R.L., London, E.D. (1995). Cerebral glucose utilization in polysubstance abuse. Neuropsychopharmacology, 13, 22-31.

Steen, R.G., Ogg, R.J., Reddick, W.E. y Kingsley, P.B. (1997). Age related changes in the pediatric brain: Quantitive MR evidence of maturational changes during adolescence. American Journal of Neuroradiology, 18, 819-828.

Steinberg, M.A., Kosten, T.A. y Rounsaville, B.J. (1992). Cocaine abuse and pathological gambling. American Journal of Addictions, 1, 121-132.

Strand, J.G. y Benjamin, L.S. (1997). Resistance to change in individuals with personality disorder. Current Opinions in Psychiatry, 10, 132-135.

Strang, J. y Gurling, H. (1989). Computerized tomography and neuropsychological associations in long-term heroin addicts. Addiction, 85, 1011-1019.

Strickland, T.L., Mena, I., Villanueva-Meyer, J., Cummings, J., Mehringer, C.M., Satz, P. y Myers, H. (1993). Cerebral perfusion and neuropsychological consequences of chronic cocaine use. Journal of Neuropsychiatry, 5 , 419-437.

Strickland, T.L. y Stein, R. (1995). Cocaine induced cerebrovascular impairment: Challenges to neuropsychological assessment. Neuropsychology Review, 5, 69-79.

Strickland, T.L., Miller, B.L., Kowell, A. y Stein, R. (1998). Neurobiology of cocaine-induced organic brain impairment: contributions from functional neuroimaging. Neuropsychology Review, 8 (1), 1-8.

Tapert, S.F. y Brown, S.A. (1999). Neuropsychological correlates of adolescent substance abuse: Four years outcomes. Journal of the International Neuropsychological Society, 5, 481-495.

Tarter, R., Jacob, T. y Bremmer, B. (1989). Specific cognitive impairment in sons of early onset alcoholics. Alcohol: Clinical and Experimental Research, 13, 786-789.

Tarter, R.E., Mezzich, A.C., Hsieh, Y. y Parks, S.M. (1995). Cognitive capacity in female adolescent substance abusers. Drug and Alcohol Dependence, 39, 15-21.

Tracy, J.I., Josiassen, R.C. y Bellack, A.S. (1995). Neuropsychology of dual diagnosis:

understanding rhe combined effects of schizophrenia and substance use disorders. Clinical Psychology Review, 15(2), 67-97.

Tunving, K., Lundqvist, T. y Ericsson, D. (1988). Marihuana: An international research. En : G. Chesher, P. Consroe y R.E. Musty (eds), 207-212. Australian Government Publishing Service: Canberra.

Tunving, K., Thulin, S.O., Risberg, J. y Warkentin, J. (1985). Psychiatry Research, 17, 15-21.

Van Gorp, W.G., Wilkins, J.N., Hinkin, C.H., Moore, L.H., Hull, J., Horner, M.D. y Plotkin, D. (1999). Declarative and procedural memory functioning in abstinent cocaine abusers. Archives of General Psychiatry, 56, 85-89.

Varma, V.K., Malhotra, A.K., Dang, R., Das, K. Y Nehra, R. (1988). Cannabis and cognitive function: a prospective study. Drug and Alcohol Dependence, 21, 147-152.

Verheul, R., Brink, W. y Geerlings, P.J. (1999). A three pathway biopsychological model of craving for alcohol. Alcohol Alcohol, 34, 347-378.

Verheul, R. y Brink, W. (2000). The role of personality pathology in the ethiology and treatment of substance use disorders. Currents Opinions in Psychiatry, 13, 30.

Verheul, R. (2001). Co-morbidity of personality disorders in individuals with substance use disorders. European Psychiatry, 16, 274-282.

Volkow, N.D., Mullani, N., Gould, K.L., Adler, S., y Krajewsky, K. (1988). Cerebral blood flow in chronic cocaine users: a study with positron emission tomography. British Journal of Psychiatry, 152, 641-148.

Volkow, N.D., Fowler, J.S., Wolf, A.P., Cchyler, D., Shiue, C.Y., Alpert, R. et al. (1990). Effects of chronic cocaine abuse on postsynaptic dopamine receptors. American Journal of Psychiatry, 147, 719-724.

Volkow, N.D., Fowler, J.S., Wolf, A.P., Hitzemann, R., Dewey, S., Bendriem, B., Alpert, R.y Hoff, A. (1991). Changes in brain glucose metabolism in cocaine dependence and withdrawal. American Journal of Psychiatry, 148, 621-626.

Volkow, N.D., Gillespie, H., Mullani, N., Tancredi, L., Grant, C., Valentine, A. y Hollister, L. (1996). Brain glucose metabolism in chronic marijuana users at baseline and during marijuana intoxication. Psychiatry Research: Neuroimaging, 67, 29-38.

Volkow, N.D., Wang, G.J., Fowler, J.S., Logan, J., Gatley, S.J., Hitzeman, R., Chen, A.D., Dewey, S.L. y Pappas, N. (1997). Decreased striatal dopaminergic 
responsiveness in detoxified cocaine-dependent subjects. Nature, 386, 830-833.

Volkow, N.D. y Fowler, J.S. (2000). Addiction: a disease of compulsion and drive: involvement of the orbitofrontal cortex. Cerebral Cortex, 10, 318-325.

Vollenweider, F.X., Maguire, R.P., Leenders, K.L., Mathis, K. y Angst, J. (1998). Effects of high anphetamine dose on mood and cerebral glucose metabolism in normal volunteers using positron emission tomography (PET). Psychiatry Research: Neuroimaging, 83, 149-162.

Wagner, M.T. y Cusham, L.A. (1994). Neuroanatomic and neuropsychological predictors of unawareness of cognitive deficits in the vascular population. Archives of Clinical Neuropsychology, 9, 57-69.

Wareing, M., Fisk, J.E. y Murphy, P.N. (2000). Working memory deficits in current and previous users of MDMA ("ecstasy"). British Journal of Psychology, 91, 181-188.

Washton A.M. y Gold, M.S. (1984). Chronic cocaine abuse: Evidence and adverse effects on health and functioning. Psychiatric Annals, 17, 733-743.

Weiss, R.D., Griffin, M.L. y Mirin, S.M. (1992). Drug abuse as self-medication for depression: An empirical study. American Journal of Drug and Alcohol Abuse, 18 (2), 121-129.

Wiesbeck, G. y Taeschner, K.L. (1991)A cerebral computed tomography study of patients with drug-induced psychoses. European Archives of Psychiatry and Clinical Neuroscience, 241, 88-90.
Wig, N.N. y Varma, V.K. (1977). Pattern of long term heavy cannabis use in North India and its effects on cognitive functions: a preliminary report. Drug and Alcohol Dependence, 2, 211-219.

Wilson, J., Levey, A., Bergeron, C., Kalasinsky, K., Ang, L., Peretty, F., Adams, V.I., Smialek, J., Anderson, W.R., Shannak, K., Deck, J., Niznik, H.B. y Kish, S.J. (1996). Striatal dopamine, dopamine transporter, and vesicular monoamine transporter in chronic cocaine users. Annals of Neurology, 40 (3), 428-439.

Wilson, W., Mathew, R., Turkington, T., Hawk, T., Coleman, R.E. y Provenzale, J. (2000). Brain morphological changes and early marijuana use: a magnetic resonance and positron emission tomography study. Journal of Addictive Diseases, 19, 1-22.

Yuille, J.C., Tollestrup, P.A., Marxsen, D., Porter, S. Y Herve, H.M.F. (1998). An exploration of the effects of marijuana on eyewitness memory. International Journal of Law and Psychiatry, 21 (1), 117-128.

Yuspeh, R., Selby, M.J. y Ririe, J. (1992). Neuropsychological functioning among long-term substances abusers with varying levels of abstinence. Clinical Neuropsychology, 6, 334 .

Zimmerman, M. y Coryell, W. (1989). DSM-III personality disorder diagnosis in a nonpatient sample: demographic correlates and comorbidity. Archives of General Psychiatry, 46, 682-689.

Zuckerman, M. (1999). Vulnerability to psychopathology: a biosocial model. Washington DC: American Psychological Association. 